TRANSACTIONS OF THE

AMERICAN MATHEMATICAL SOCIETY

Volume 362, Number 8, August 2010, Pages 3963-3996

S 0002-9947(10)04985-8

Article electronically published on March 17, 2010

\title{
ON A SYMPLECTIC GENERALIZATION OF PETRIE'S CONJECTURE
}

\author{
SUSAN TOLMAN
}

\begin{abstract}
Motivated by the Petrie conjecture, we consider the following questions: Let a circle act in a Hamiltonian fashion on a compact symplectic manifold $(M, \omega)$ which satisfies $H^{2 i}(M ; \mathbb{R})=H^{2 i}\left(\mathbb{C P}^{n}, \mathbb{R}\right)$ for all $i$. Is $H^{j}(M ; \mathbb{Z})=H^{j}\left(\mathbb{C P}^{n} ; \mathbb{Z}\right)$ for all $j$ ? Is the total Chern class of $M$ determined by the cohomology ring $H^{*}(M ; \mathbb{Z})$ ? We answer these questions in the sixdimensional case by showing that $H^{j}(M ; \mathbb{Z})$ is equal to $H^{j}\left(\mathbb{C P}^{3} ; \mathbb{Z}\right)$ for all $j$, by proving that only four cohomology rings can arise, and by computing the total Chern class in each case. We also prove that there are no exotic actions. More precisely, if $H^{*}(M ; \mathbb{Z})$ is isomorphic to $H^{*}\left(\mathbb{C P}^{3} ; \mathbb{Z}\right)$ or $H^{*}\left(\widetilde{G}_{2}\left(\mathbb{R}^{5}\right) ; \mathbb{Z}\right)$, then the representations at the fixed components are compatible with one of the standard actions; in the remaining two cases, the representation is strictly determined by the cohomology ring. Finally, our results suggest a natural question: Do the remaining two cohomology rings actually arise? This question is closely related to some interesting problems in symplectic topology, such as embeddings of ellipsoids.
\end{abstract}

\section{INTRODUCTION}

In the early 1970s, Ted Petrie wanted to address two related fundamental questions: Given a compact Lie group $G$ and a manifold $M$, does $M$ admit a $G$-action:1 If so, how many different actions can we find?

One of his important insights was that these questions are much more tractable when $M$ is a homotopy projective space, that is, a simply connected manifold so that $H^{*}(M ; \mathbb{Z})=H^{*}\left(\mathbb{C P}^{n} ; \mathbb{Z}\right)$ as rings, or equivalently a manifold which is homotopy equivalent to $\mathbb{C P}^{n}$.

For example, the first key step in answering these questions is understanding the relationship between the tangent bundle near the fixed components and the global invariants of $M$. Petrie proved that if the circle acts on a homotopy projective space with isolated fixed points, then the Pontrjagin classes are determined by the representations at the fixed points $[\mathrm{Pe}$ ].

Motivated by this and other evidence, he stated what is now known as the Petrie conjecture: If a homotopy projective space $M$ admits a circle action, then the Pontrjagin classes of $M$ are standard, that is, agree with the Pontrjagin classes of $\mathbb{C P}^{n}$ itself. Although this conjecture has not been resolved in general, it has motivated a good deal of research. In particular, it has been proven if $M$ is at most

Received by the editors September 19, 2007.

2000 Mathematics Subject Classification. Primary 53D20.

The author was partially supported by National Science foundation grant DMS \#07-07122.

${ }^{1}$ We shall always assume that our actions are non-trivial. 
eight-dimensional Dej, Ja, if $M$ admits an invariant almost complex structure whose first Chern class is at least $\frac{1}{2} \operatorname{dim}(M)+1$ times the generator of $H^{2}(M ; \mathbb{Z})$ $[\mathrm{Ha}$, and in many other special cases [Des, $\mathrm{Ma}, \mathrm{Mu}, \mathrm{Pe} 2, \mathrm{TsWa}, \mathrm{Wan}, \mathrm{Yo}$.

We are interested in addressing the analogous questions for symplectic manifolds: Given a compact Lie group $G$ and a symplectic manifold $(M, \omega)$, does $M$ admit a Hamiltonian $G$-action? If so, how many different actions can we find?

In the symplectic case, several additional tools are available. For example, there is an almost complex structure $J: T(M) \longrightarrow T(M)$ which is compatible with $\omega$, i.e., $\omega(J \cdot, \cdot)$ is a Reimannian metric. Moreover, the set of such structures is contractible, and so there is a well-defined total Chern class $c(M) \in H^{*}(M ; \mathbb{Z})$. Additionally, the components of the moment map $\Phi: M \longrightarrow \mathfrak{g}^{*}$ are Morse-Bott functions with extremely nice properties; see $\$ 2$.

Therefore, instead of insisting that our symplectic manifold $(M, \omega)$ be a homotopy projective space, we merely assume that $H^{2 i}(M ; \mathbb{R})=H^{2 i}\left(\mathbb{C P}^{n} ; \mathbb{R}\right)$ for all $i$. We prove that if the circle acts on such a manifold in a Hamiltonian fashion with isolated fixed points, then both the cohomology ring and the total Chern class are determined by the representations at the fixed points; see Corollary 3.16 and Remark 3.17. This leads to the following questions.

Question 1. Consider a Hamiltonian circle action on a symplectic manifold $(M, \omega)$ which satisfies $H^{2 i}(M ; \mathbb{R})=H^{2 i}\left(\mathbb{C P}^{n} ; \mathbb{R}\right)$ for all $i$. Is $H^{j}(M ; \mathbb{Z})=H^{j}\left(\mathbb{C P} \mathbb{P}^{n} ; \mathbb{Z}\right)$ for all $j$ ? Is the total Chern class $c(M)$ completely determined by the cohomology ring $H^{*}(M ; \mathbb{Z})$ ?

Our first main theorem answers this question affirmatively in the 6-dimensional case. In fact, we are able to show that only a few possible rings arise.

Theorem 1. Let the circle act on a 6-dimensional compact symplectic manifold $(M, \omega)$ with moment map $\Phi: M \longrightarrow \mathbb{R}$. If $H^{2}(M, \mathbb{R})=\mathbb{R}$, then one of the following four statements is true:

(A) $H^{*}(M ; \mathbb{Z})=\mathbb{Z}[x] /\left(x^{4}\right)$ and $c(M)=1+4 x+6 x^{2}+4 x^{3}$.

(B) $H^{*}(M ; \mathbb{Z})=\mathbb{Z}[x, y] /\left(x^{2}-2 y, y^{2}\right)$ and $c(M)=1+3 x+8 y+4 x y$.

(C) $H^{*}(M ; \mathbb{Z})=\mathbb{Z}[x, y] /\left(x^{2}-5 y, y^{2}\right)$ and $c(M)=1+2 x+12 y+4 x y$.

(D) $H^{*}(M ; \mathbb{Z})=\mathbb{Z}[x, y] /\left(x^{2}-22 y, y^{2}\right)$ and $c(M)=1+x+24 y+4 x y$.

In each case, $x$ has degree 2 and $y$ has degree 4 .

Remark 1.1. Since $H^{2}(M ; \mathbb{R})=\mathbb{R}$ and $M$ is a 6 -dimensional symplectic manifold, Poincaré duality implies that $b_{0}=b_{2}=b_{4}=b_{6}=1$, where $b_{i}=\operatorname{dim}\left(H^{i}(M ; \mathbb{R})\right)$ denotes the $i$ th Betti number. If the fixed set is discrete, then this immediately implies that $H^{j}(M ; \mathbb{Z})=H^{j}\left(\mathbb{C P}^{3} ; \mathbb{Z}\right)$ for all $j$, that is, that $b_{1}=b_{3}=b_{5}=0$ and the cohomology is torsion free; see $\oint_{2}$. However, when the fixed set is not discrete, this fact is somewhat surprising; it follows from the analysis in $₫$

Remark 1.2. By Corollary 1.6 below, the manifold $M$ described above is simply connected. If we assume that statement (A) is true, then $w_{2}(M)=0$; therefore, Wall's theorem implies that $M$ and $\mathbb{C P}^{3}$ are diffeomorphic Wal. Similarly, any two manifolds which satisfy statement (C) must be diffeomorphic.

Petrie was able to construct exotic circle actions on projective spaces, that is, actions so that the induced representations at the fixed points do not agree with those of any circle subgroup $S^{1} \subset \mathrm{SU}(n+1)$. Our second main theorem is that, in 
contrast, in the 6-dimensional symplectic case, there are no exotic actions. More precisely, the representations at the fixed components are either strictly determined by the cohomology ring or are compatible with one of the standard actions described below.

Example 1.3. Given $n>1$, let $\mathbb{C P}^{n}$ denote the projective space of lines in $\mathbb{C}^{n+1}$. Since this $2 n$-dimensional manifold naturally arises as a coadjoint orbit of $\mathrm{SU}(n+1)$, it inherits a symplectic form $\omega$ and a Hamiltonian $\mathrm{SU}(n+1)$ action. Hence, every circle subgroup $S^{1} \subset \mathrm{SU}(n+1)$ induces a Hamiltonian circle action on $\mathbb{C P}^{n}$.

Example 1.4. Given $n>1$, let $\widetilde{G}_{2}\left(\mathbb{R}^{2 n+1}\right)$ denote the Grassmannian of oriented 2 -planes in $\mathbb{R}^{2 n+1}$. Since this $(4 n-2)$-dimensional manifold naturally arises as a coadjoint orbit of $\mathrm{SO}(2 n+1)$, it inherits a symplectic form $\omega$ and a Hamiltonian $\mathrm{SO}(2 n+1)$ action. Hence, every circle subgroup $S^{1} \subset \mathrm{SO}(2 n+1)$ induces a Hamiltonian circle action on $\widetilde{G}_{2}\left(\mathbb{R}^{2 n+1}\right)$.

Given any subgroup $H \subset S^{1}$, let $M^{H}$ denote the set of points fixed by $H$. Each component $N \subset M^{H}$ is a symplectic manifold which inherits a symplectic circle action with moment map $\left.\Phi\right|_{N}$. If $H \neq\{e\}$, we call each component $N$ of $M^{H}$ which is not fixed by $S^{1}$ an isotropy submanifold. Each two-dimensional isotropy submanifold is a sphere which contains exactly two isolated fixed points; we call these isotropy spheres. We can now state our second main theorem, which is an immediate consequence of Propositions 4.1, 6.1 and 7.1, and the remarks subsequent to each.

Theorem 2. Let the circle act faithfully 2 on a 6-dimensional compact symplectic manifold $(M, \omega)$ with moment map $\Phi: M \longrightarrow \mathbb{R}$. If $H^{2}(M, \mathbb{R})=\mathbb{R}$, then one of the following four statements is true 3

(A) There is a subgroup $S^{1} \subset \mathrm{SU}(4)$ and an orientation-preserving diffeomorphism $f: M^{S^{1}} \longrightarrow\left(\mathbb{C P}^{3}\right)^{S^{1}}$ so that $\left.T(M)\right|_{M^{S^{1}}} \cong f^{*}\left(\left.T\left(\mathbb{C P}^{3}\right)\right|_{\left(\mathbb{C P}^{3}\right)^{S^{1}}}\right)$.

(B) There is a subgroup $S^{1} \subset \mathrm{SO}(5)$ and an orientation-preserving diffeomorphism $f: M^{S^{1}} \longrightarrow \widetilde{G}_{2}\left(\mathbb{R}^{5}\right)^{S^{1}}$ so that $\left.T(M)\right|_{M^{S^{1}}} \cong f^{*}\left(\left.T\left(\widetilde{G}_{2}\left(\mathbb{R}^{5}\right)\right)\right|_{\widetilde{G}_{2}\left(\mathbb{R}^{5}\right)^{S^{1}}}\right)$.

(C) The fixed set consists of four points; the weights at these points are

$$
\{1,2,3\},\{1,-1,4\},\{1,-1,-4\} \text {, and }\{-1,-2,-3\} \text {. }
$$

(D) The fixed set consists of four points; the weights at these points are

$$
\{1,2,3\},\{1,-1,5\},\{1,-1,-5\} \text {, and }\{-1,-2,-3\} \text {. }
$$

Moreover, $M$ contains a pair of isotropy spheres which intersect in two points in cases $(C)$ and $(D)$, but not in cases $(A)$ or $(B)$.

Remark 1.5. In case (A), $M$ is cobordant to $\mathbb{C P}^{3}$ (with some multiple of the standard symplectic form) as a stable-complex Hamiltonian $G$-space. In fact, we may assume that $f$ is a symplectomorphism; see Remarks 3.17 and 4.3 Similar comments apply in each case.

${ }^{2}$ A group $G$ acts faithfully on $M$ if for every non-trivial $g \in G$ there exists $m \in M$ so that $g \cdot m \neq m$.

3 Throughout this paper, the symbol $\cong$ implies that the two sides are equivariantly isomorphic complex vector bundles. 
We can convert any non-trivial circle action into an effective circle action by quotienting out the subgroup which acts trivially. Therefore, Theorem 1 follows immediately from Theorem 2 above and Corollaries 3.16 and 3.19, see Example 3.22.

In each of the cases described above, each component of the fixed set is simply connected. By [Li1, this implies that Theorem 2 has the following corollary.

Corollary 1.6. Let the circle act faithfully on a 6-dimensional compact symplectic manifold $(M, \omega)$ with moment map $\Phi: M \longrightarrow \mathbb{R}$. If $H^{2}(M, \mathbb{R})=\mathbb{R}$, then $M$ is simply connected.

Finally, we can describe the equivariant cohomology of $M$; by definition, this is $H_{S^{1}}^{*}(M)=H^{*}\left(M \times_{S^{1}} S^{\infty}\right)$. For example, if $p$ is a point, then $H_{S^{1}}^{*}(p ; \mathbb{Z})=$ $H^{*}\left(\mathbb{C P} \mathbb{P}^{\infty} ; \mathbb{Z}\right)=\mathbb{Z}[t]$. The projection map $\pi: M \times_{S^{1}} S^{\infty} \longrightarrow \mathbb{C P} \mathbb{P}^{\infty}$ induces a pullback map

$$
\pi^{*}: H^{*}\left(\mathbb{C P}^{\infty} ; \mathbb{Z}\right) \longrightarrow H_{S^{1}}^{*}(M ; \mathbb{Z})
$$

thus, $H_{S^{1}}^{*}(M ; \mathbb{Z})$ is a $H^{*}\left(\mathbb{C P}^{\infty} ; \mathbb{Z}\right)$ module. Moreover, the inclusion $\iota: M^{S^{1}} \longrightarrow M$ induces a restriction map $\iota^{*}: H_{S^{1}}^{*}(M ; \mathbb{Z}) \longrightarrow H_{S^{1}}^{*}\left(M^{S^{1}} ; \mathbb{Z}\right)$; define

$$
\left.H_{S^{1}}^{*}(M ; \mathbb{Z})\right|_{M^{S^{1}}}=\iota^{*}\left(H_{S^{1}}^{*}(M ; \mathbb{Z})\right) .
$$

Finally, let $c^{S^{1}}(M) \in H_{S^{1}}^{*}(M ; \mathbb{Z})$ denote the total equivariant Chern class of $M$.

The theorem below follows immediately from Theorem 2 and Corollaries 3.13 and 3.14, see Example 3.15. Note that, since $H^{*}\left(M^{S^{1}} ; \mathbb{Z}\right)$ has no torsion, the image $\left.H_{S^{1}}^{*}(M ; \mathbb{Z})\right|_{M^{S^{1}}}$ naturally determines the equivariant cohomology ring itself; see $₫ 2$,

Theorem 3. Let the circle act faithfully on a 6-dimensional compact symplectic manifold $(M, \omega)$ with moment map $\Phi: M \longrightarrow \mathbb{R}$. If $H^{2}(M, \mathbb{R})=\mathbb{R}$, then one of the following four statements is true:

(A) There is a subgroup $S^{1} \subset \mathrm{SU}(4)$ and diffeomorphism $f: M^{S^{1}} \longrightarrow\left(\mathbb{C P}^{3}\right)^{S^{1}}$ so that

$$
\begin{aligned}
& \left.H_{S^{1}}^{*}(M ; \mathbb{Z})\right|_{M^{S^{1}}}=f^{*}\left(\left.H_{S^{1}}^{*}\left(\mathbb{C P}^{3} ; \mathbb{Z}\right)\right|_{\left(\mathbb{C P}^{3}\right)^{S^{1}}}\right) \text { and } \\
& \left.c^{S^{1}}(M)\right|_{M^{S^{1}}}=f^{*}\left(\left.c^{S^{1}}\left(\mathbb{C P}^{3}\right)\right|_{\left(\mathbb{C P}^{3}\right)^{S^{1}}}\right) .
\end{aligned}
$$

(B) There is a subgroup $S^{1} \subset \mathrm{SO}(5)$ and diffeomorphism $f: M^{S^{1}} \longrightarrow \widetilde{G}_{2}\left(\mathbb{R}^{5}\right)^{S^{1}}$ so that

$$
\begin{aligned}
& \left.H_{S^{1}}^{*}(M ; \mathbb{Z})\right|_{M^{S^{1}}}=f^{*}\left(\left.H_{S^{1}}^{*}\left(\widetilde{G}_{2}\left(\mathbb{R}^{5}\right) ; \mathbb{Z}\right)\right|_{\widetilde{G}_{2}\left(\mathbb{R}^{5}\right)^{S^{1}}}\right) \text { and } \\
& \left.c^{S^{1}}(M)\right|_{M^{S^{1}}}=f^{*}\left(\left.c^{S^{1}}\left(\widetilde{G}_{2}\left(\mathbb{R}^{5}\right)\right)\right|_{\widetilde{G}_{2}\left(\mathbb{R}^{5}\right)^{S^{1}}}\right) .
\end{aligned}
$$

(C) The fixed set consists of four points: $p_{0}, p_{1}, p_{2}$ and $p_{3}$. As an $H^{*}\left(\mathbb{C} \mathbb{P}^{\infty} ; \mathbb{Z}\right)=$ $\mathbb{Z}[t]$ module, $H_{S^{1}}^{*}(M ; \mathbb{Z})$ is generated by $1, \alpha_{1}, \alpha_{2}$, and $\alpha_{3}$, where

$$
\begin{gathered}
\left.\alpha_{1}\right|_{p_{1}}=t,\left.\alpha_{1}\right|_{p_{2}}=5 t,\left.\alpha_{1}\right|_{p_{3}}=6 t,\left.\alpha_{2}\right|_{p_{2}}=4 t^{2}, \\
\left.\alpha_{2}\right|_{p_{3}}=6 t^{2},\left.\alpha_{3}\right|_{p_{3}}=6 t^{3}, \text { and }\left.\alpha_{i}\right|_{p_{j}}=0 \forall j<i ; \text { moreover }, \\
\left.c^{S^{1}}(M)\right|_{p_{0}}=1+6 t+11 t^{2}+6 t^{3},\left.\quad c^{S^{1}}(M)\right|_{p_{1}}=1+4 t-t^{2}-4 t^{3}, \\
\left.c^{S^{1}}(M)\right|_{p_{2}}=1-4 t-t^{2}+4 t^{3} \text { and }\left.c^{S^{1}}(M)\right|_{p_{3}}=1-6 t+11 t^{2}-6 t^{3} .
\end{gathered}
$$


(D) The fixed set consists of four points: $p_{0}, p_{1}, p_{2}$ and $p_{3}$. As an $H^{*}(\mathbb{C P} ; \mathbb{Z})=$ $\mathbb{Z}[t]$ module, $H_{S^{1}}^{*}(M ; \mathbb{Z})$ is generated by $1, \alpha_{1}, \alpha_{2}$, and $\alpha_{3}$, where

$$
\begin{gathered}
\left.\alpha_{1}\right|_{p_{1}}=t,\left.\alpha_{1}\right|_{p_{2}}=6 t,\left.\alpha_{1}\right|_{p_{3}}=12 t,\left.\alpha_{2}\right|_{p_{2}}=5 t^{2}, \\
\left.\alpha_{2}\right|_{p_{3}}=6 t^{2},\left.\alpha_{3}\right|_{p_{3}}=6 t^{3}, \quad \text { and }\left.\alpha_{i}\right|_{p_{j}}=0 \forall j<i ; \text { moreover, } \\
\left.c^{S^{1}}(M)\right|_{p_{0}}=1+6 t+11 t^{2}+6 t^{3},\left.\quad c^{S^{1}}(M)\right|_{p_{1}}=1+5 t-t^{2}-5 t^{3}, \\
\left.c^{S^{1}}(M)\right|_{p_{2}}=1-5 t-t^{2}+5 t^{3}, \quad \text { and }\left.c^{S^{1}}(M)\right|_{p_{3}}=1-6 t+11 t^{2}-6 t^{3} .
\end{gathered}
$$

Note that, in the case that the action is semifree and there is no four-dimensional fixed component, these three theorems are due to $\mathrm{Li}[\mathrm{Li} 2$.

Open questions. In these theorems, the first two cases correspond to Examples 1.3 and 1.4 but the last two cases do not correspond to any known examples 4 This raises the following natural question:

Question 2. Do there exist examples exhibiting properties (C) or (D) of Theorem 2 ? More precisely, let $l=4$ or 5 . Does there exist a Hamiltonian circle action on a compact symplectic (alternatively, Kähler) manifold so that the fixed set consists of four points with weights $\{1,2,3\},\{1,-1, l\},\{1,-1,-l\}$, and $\{-1,-2,-3\}$ ?

We do not know the answer to this question. These manifolds cannot be ruled out by any of the techniques used in this paper; see also Remarks 2.10 and 2.11 .

Moreover, this question seems to be related to interesting problems in symplectic topology. To see this, we need to introduce some more notation. Given an $(n+1)$ tuple of natural numbers $\mathbf{k}=\left(k_{0}, \ldots, k_{n}\right)$, consider the weighted projective space of type $\mathbf{k}$,

$$
\mathbb{C P}^{n}(\mathbf{k})=S^{2 n+1} /\left(z_{0}, \ldots, z_{n}\right) \sim\left(\lambda^{k_{0}} z_{0}, \ldots, \lambda^{k_{n}} z_{n}\right) ;
$$

let $\overline{\mathbb{C P}}^{n}(\mathbf{k})$ denote the same manifold with the opposite orientation. Let $\alpha_{\mathbf{k}} \in$ $H^{2}\left(\mathbb{C P}^{n}(\mathbf{k})\right)$ denote the first Chern class of the tautological circle bundle $S^{2 n+1} \longrightarrow$ $\mathbb{C P}^{n}(\mathbf{k})$. Finally, given real numbers $a$ and $b$, define the ellipsoid

$$
E(a, b)=\left\{\left(x_{1}, x_{2}\right) \in \mathbb{C}^{2} \mid \frac{1}{a} x_{1}^{2}+\frac{1}{b} x_{2}^{2} \leq 1\right\} .
$$

Now suppose that a manifold $(M, \omega)$ satisfying the conditions of Question 2 does exist. By Corollary 3.13 (see Example 3.15) and Lemma 2.7 - after possibly rescaling $\omega$ - there exists a moment map $\Phi: M \longrightarrow \mathbb{R}$ so that

$$
\Phi\left(p_{0}\right)=-6, \Phi\left(p_{1}\right)=-l, \Phi\left(p_{2}\right)=l, \text { and } \Phi\left(p_{3}\right)=6,
$$

where $p_{i}$ is the unique fixed point of index $2 i$ for all $i$ such that $0 \leq 2 i \leq 6$. By [Go], this implies that for all $\kappa \in(-l, l)$, the reduced space $M_{\kappa}=\Phi^{-1}(\kappa) / S^{1}$ is diffeomorphic to the connected sum

$$
X=\mathbb{C P}^{2}(1,2,3) \# \overline{\mathbb{C P}}^{2}(1,1, l) .
$$

Moreover, let $\omega_{\kappa} \in \Omega^{2}\left(M_{\kappa}\right)$ denote the reduced symplectic form; the cohomology class $\left[\omega_{\kappa}\right] \in H^{2}\left(M_{\kappa}\right)$ is the unique class so that $\left.\left[\omega_{\kappa}\right]\right|_{\mathbb{C P}^{1}(2,3)}=(6+\kappa) \alpha_{(2,3)}$ and $\left.\left[\omega_{\kappa}\right]\right|_{\overline{C P}^{1}(1, l)}=-(l+\kappa) \alpha_{(1, l)}$. Here, the inclusions of $\mathbb{C P}^{1}(2,3)$ and $\mathbb{C P}^{1}(1, l)$ into $X=M_{\kappa}$ are induced by the natural inclusions $\mathbb{C P}^{1}(2,3) \subset \mathbb{C P}^{2}(1,2,3)$ and

\footnotetext{
${ }^{4}$ Since this paper was originally submitted, McDuff has used symplectic techniques to construct manifolds corresponding to the last two cases Mc2. In fact, as she points out, both manifolds (which are Kähler) were already known.
} 
$\overline{\mathbb{C P}}^{1}(1, l) \subset \overline{\mathbb{C P}}^{2}(1,1, l)$. In particular, an affirmative answer to Question 2 implies an affirmative answer to the question below.

Question 3. Given any $\lambda<2$, is there a symplectic (Kähler) form

$$
\begin{gathered}
\omega_{\lambda} \in \Omega^{2}\left(\mathbb{C P}^{2}(1,2,3) \# \overline{\mathbb{C P}}^{2}(1,1, l)\right) \text { such that } \\
{\left.\left[\omega_{\lambda}\right]\right|_{\mathbb{C P}^{1}(2,3)}=(6+l) \alpha_{(2,3)} \text { and }\left.\left[\omega_{\lambda}\right]\right|_{\overline{\mathbb{P}}^{1}(1, l)}=-\lambda l \alpha_{(1, l)} ?}
\end{gathered}
$$

As in the manifold case, we can construct such a symplectic form if we can find the symplectic embeddings described below.

Question 4. Given any $\lambda<2$, is there a symplectic embedding

$$
E(\lambda, \lambda l) \hookrightarrow E\left(\frac{6+l}{3}, \frac{6+l}{2}\right) ?
$$

Unfortunately, although symplectic embeddings have been extensively studied, this particular question does not seem to follow easily from known results $[\mathrm{S}] 5$ In particular, it cannot be ruled out by volume constraints; $\frac{\operatorname{volume}(E(2,2 l))}{\operatorname{volume}\left(E\left(\frac{6+2}{3}, \frac{6+l}{2}\right)\right)}$ is equal to $\frac{24}{25}$ if $l=4$ and to $\frac{120}{121}$ if $l=5$.

We conclude this section with a brief overview of this paper. In 42 we introduce some background material and establish our notation. In $\$ 3$, we prove a few useful results which hold in arbitrary dimensions. As a consequence, we prove that Theorem 1 and Theorem 3 follow immediately from Theorem 2. In \$4 we return to the six-dimensional case, proving Theorem 2 in the case that the fixed set is not discrete. We spend the remainder of the paper proving this theorem in the case that the fixed set is discrete. To do so, we first define a labeled multigraph associated to $M$ in $\$ 5$ and then prove Theorem 2 in the cases that the associated multigraph is simple and not simple in $\$ 6$ and $\$ 7$ respectively.

\section{ACKNOWLEDGMENTS}

The author would like to thank Jonathan Weitsman for inspiring these results by introducing her to the Petrie conjecture. The author would also like to thank Yael Karshon and Dusa McDuff for useful discussions, and the referee for suggesting many improvements to the exposition.

\section{BACKGROUND}

In this section, we introduce some background material and establish our notation.

Let $M$ be a compact manifold. A symplectic form on $M$ is a closed, nondegenerate two-form $\omega \in \Omega^{2}(M)$. A circle action on $M$ is symplectic if it preserves $\omega$. A symplectic circle action is Hamiltonian if there exists a moment map, that is, a map $\Phi: M \longrightarrow \mathbb{R}$ such that

$$
-d \Phi=\iota_{\xi_{M}} \omega
$$

where $\xi_{M}$ is the vector field on $M$ induced by the circle action. Since $\iota_{\xi_{M}} \omega$ is closed, every symplectic action is Hamiltonian if $H^{1}(M ; \mathbb{R})=0$.

Let the circle act on a compact symplectic manifold $(M, \omega)$ with moment map $\Phi: M \longrightarrow \mathbb{R}$. Since the set of compatible almost complex structures $J: T(M) \longrightarrow$

${ }^{5}$ Again, McDuff has published new results on this question since this paper was originally submitted [Mc1]; she uses these in [Mc2]. 
$T(M)$ is contractible, there is a well-defined multiset of integers, called weights, associated to each fixed point $p$. Indeed, for any fixed component $F$, the tangent bundle $\left.T(M)\right|_{F}$ naturally splits into subbundles - one corresponding to each weight.

The moment map $\Phi: M \longrightarrow \mathbb{R}$ is a Morse-Bott function whose critical set is the fixed point set. Moreover, the negative normal bundle at $F$ is the sum of the subbundles of $\left.T(M)\right|_{F}$ with negative weights. In particular, the index of a fixed component $F$ is $2 \lambda_{F}$, where $\lambda_{F}$ is the number of negative weights in $T_{p} M$ for any $p \in F$ (counted with multiplicity). More interestingly, let $e_{S^{1}}\left(N_{F}^{-}\right) \in H_{S^{1}}^{2 \lambda_{F}}(F)$ denote the equivariant Euler class of the negative normal bundle at $F$. If $p \in M^{S^{1}}$ is an isolated point, then $e_{S^{1}}\left(N_{p}^{-}\right)=\Lambda_{p}^{-} t^{\lambda_{p}}$, where $\Lambda_{p}^{-} \in \mathbb{Z} \backslash\{0\}$ is the product of the negative (integer) weights at $p$. More generally, for any fixed component $F$, we can naturally identify $H_{S^{1}}^{*}(F)=H^{*}\left(F \times \mathbb{C P}^{\infty}\right)$ with $H^{*}(F)[t]$. Under this identification, $e_{S^{1}}\left(N_{F}^{-}\right)$is a polynomial in $t$; the highest degree term is $\Lambda_{F}^{-} t^{\lambda_{F}}$. Therefore, as Atiyah and Bott pointed out, $e_{S^{1}}\left(N_{F}^{-}\right)$is not a zero divisor in $H_{S^{1}}^{2 \lambda_{F}}(F ; \mathbb{Q})$ for any fixed component $F$.

Kirwan uses this idea to prove three remarkable theorems: "perfection", "injectivity", and "formality" [Ki]. Let $R=\mathbb{Z}$ if the fixed set is torsion free, that is, $H^{*}\left(M^{S^{1}} ; \mathbb{Z}\right)$ has no torsion; otherwise, let $R=\mathbb{Q}$. (See ToWe1 for comments on the integral case.) Let $F$ be any fixed component, and let $M^{ \pm}=$ $\Phi^{-1}(-\infty, \Phi(F) \pm \epsilon)$, where $\epsilon>0$ is sufficiently small. Since $e_{S^{1}}\left(N_{F}^{-}\right) \in H_{S^{1}}^{*}(F ; R)$ is not a zero divisor, the natural restriction $H_{S^{1}}^{*}\left(M^{+}, M^{-} ; R\right) \longrightarrow H_{S^{1}}^{*}(F ; R)$ is an injection. Therefore, the long exact sequence in equivariant cohomology for the pair $\left(M^{+}, M^{-}\right)$breaks into short exact sequences

$$
0 \longrightarrow H_{S^{1}}^{j}\left(M^{+}, M^{-} ; R\right) \longrightarrow H_{S^{1}}^{j}\left(M^{+} ; R\right) \longrightarrow H_{S^{1}}^{j}\left(M^{-} ; R\right) \longrightarrow 0 .
$$

If $H_{S^{1}}^{j}\left(M^{-} ; R\right)$ is a free group, this implies immediately that

$$
H_{S^{1}}^{j}\left(M^{+} ; R\right)=H_{S^{1}}^{j}\left(M^{+}, M^{-} ; R\right) \oplus H_{S^{1}}^{j}\left(M^{-} ; R\right) .
$$

By induction, $H_{S^{1}}^{j}(M ; \mathbb{R})$ is a free group and the moment map is an equivariantly perfect Morse-Bott function; in fact,

$$
H_{S^{1}}^{j}(M ; R)=\bigoplus_{F \subset M^{S^{1}}} H_{S^{1}}^{j-2 \lambda_{F}}(F ; R),
$$

where the sum is over all fixed components. Similarly, by induction and (2.1), the restriction map $\iota^{*}: H_{S^{1}}^{*}(M ; R) \longrightarrow H_{S^{1}}^{*}\left(M^{S^{1}} ; R\right)$ is an injection. Hence, every equivariant cohomology class is determined by its restriction to the fixed point set. Finally, restriction induces a natural map of exact sequences

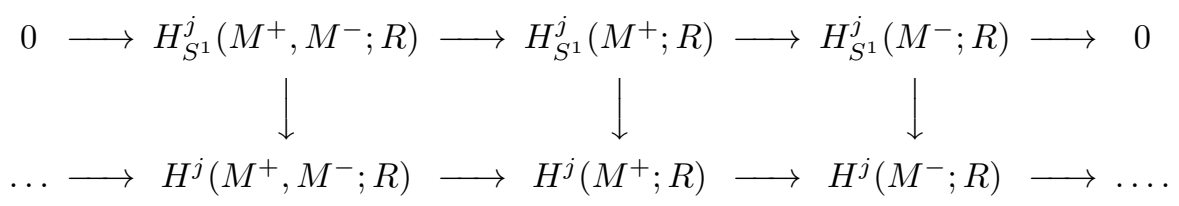

Moreover, the restriction map from $H_{S^{1}}^{*}\left(M^{+}, M^{-} ; R\right)$ to $H^{*}\left(M^{+}, M^{-} ; R\right)$ is surjective because $H_{S^{1}}^{*}(F ; R)=H^{*}(F ; R)[t]$. Hence, by an easy diagram chase, if the restriction map from $H_{S^{1}}^{*}\left(M^{-} ; R\right)$ to $H^{*}\left(M^{-} ; R\right)$ is surjective, then so is the restriction map from $H_{S^{1}}^{*}\left(M^{+} ; R\right)$ to $H^{*}\left(M^{+} ; R\right)$; moreover, the long exact sequence 
in cohomology for the pair $\left(M^{+}, M^{-}\right)$breaks into short exact sequences

$$
0 \longrightarrow H^{j}\left(M^{+}, M^{-} ; R\right) \longrightarrow H^{j}\left(M^{+} ; R\right) \longrightarrow H^{j}\left(M^{-} ; R\right) \longrightarrow 0 .
$$

By induction, $\Phi$ is a perfect Morse-Bott function and the restriction map $H_{S^{1}}^{*}(M ; R)$ $\longrightarrow H^{*}(M ; R)$ is a surjection. By Leray-Hirsch, this implies that the kernel of this map is the ideal generated by $\pi^{*}(t)$, where $t \in H^{2}\left(\mathbb{C P}^{\infty} ; R\right)$ is the generator. (See (1.7).) Hence, if we want to compute the (ordinary) cohomology of $M$, it is enough to determine the equivariant cohomology of $M$ as an $H^{*}\left(\mathbb{C P}^{\infty} ; R\right)$ module. Nearly identical arguments prove the following closely related proposition.

Proposition 2.2. Let the circle act on a compact symplectic manifold $(M, \omega)$ with moment map $\Phi: M \longrightarrow \mathbb{R}$. If the fixed set is torsion-free, let $R=\mathbb{Z}$; otherwise let $R=\mathbb{Q}$.

Given a fixed component $F$ and a class $u \in H^{i}(F ; R)$, there exists a class $\alpha \in$ $H_{S^{1}}^{i+2 \lambda_{F}}(M ; R)$ so that

(1) $\left.\alpha\right|_{F}=u e_{S^{1}}\left(N_{F}^{-}\right)$, and

(2) $\left.\alpha\right|_{F^{\prime}}=0$ for all other fixed components $F^{\prime}$ with $\Phi\left(F^{\prime}\right) \leq \Phi(F)$.

Moreover, let $\left\{u_{j}\right\}$ be a basis for $H^{*}\left(M^{S^{1}} ; R\right)$, where each $u_{j} \in H^{i_{j}}\left(F_{j} ; R\right)$ for some fixed component $F_{j}$. If $\alpha_{j} \in H_{S^{1}}^{i_{j}+2 \lambda_{F_{j}}}(M ; R)$ and $u_{j}$ satisfy (1) and (2) above for each $j$, then $\left\{\alpha_{j}\right\}$ is a basis for $H_{S^{1}}^{*}(M ; R)$ as an $H^{*}\left(\mathbb{C P} \mathbb{P}^{\infty} ; R\right)$ module.

Corollary 2.3. Let the circle act on a compact symplectic manifold $(M, \omega)$ with moment map $\Phi: M \longrightarrow \mathbb{R}$. If the fixed set is torsion-free, let $R=\mathbb{Z}$; otherwise let $R=\mathbb{Q}$. Consider $\beta \in H_{S^{1}}^{*}(M ; R)$ and $c \in \mathbb{R}$ so that $\left.\beta\right|_{F^{\prime}}=0$ for all fixed components $F^{\prime}$ such that $\Phi\left(F^{\prime}\right)<c$.

(1) If $c=\Phi(F)$ for some fixed component $F$, then $\left.\beta\right|_{F}$ is a multiple of $e_{S^{1}}\left(N_{F}^{-}\right)$.

(2) More generally, let $\left\{u_{j}\right\}$ be a basis for $H^{*}\left(M^{S^{1}} ; R\right)$, where each $u_{j} \in$ $H^{i_{j}}\left(F_{j} ; R\right)$ for some fixed component $F_{j}$. Assume that $\alpha_{j} \in H_{S^{1}}^{i_{j}+2 \lambda_{F_{j}}}(M ; R)$ and $u_{j}$ satisfy (1) and (2) above for each $j$. Then

$$
\beta=\sum_{\Phi\left(F_{j}\right) \geq c} x_{j} \alpha_{j}
$$

where $x_{j} \in H^{*}\left(\mathbb{C P}^{\infty} ; R\right)$ for all $j$. Here, the sum is over all $j$ such that $\Phi\left(F_{j}\right) \geq c$.

Proof. By the proposition above, we can write $\beta=\sum_{j} x_{j} \alpha_{j}$, where here the sum is over all $j$. If $x_{j}=0$ for all $j$ such that $\Phi\left(F_{j}\right)<c$, then the second claim holds. Moreover, if $\Phi(F)=c$ for some fixed component $F$, then properties (1) and (2) together imply that $\left.\beta\right|_{F}$ is a multiple of $e_{S^{1}}\left(N_{F}^{-}\right)$.

Otherwise, there exists $j$ so that $\Phi\left(F_{j}\right)<c$ and $x_{j} \neq 0$ but $x_{k}=0$ for $k$ such that $\Phi\left(F_{k}\right)<\Phi\left(F_{j}\right)$. By properties (1) and (2), this implies that $\left.\beta\right|_{F_{j}} \neq 0$. Since $\Phi\left(F_{j}\right)<c$, this contradicts the assumption.

The projection $\pi: M \times_{S^{1}} S^{\infty} \longrightarrow \mathbb{C P}^{\infty}$ induces a natural push-forward map $\pi_{*}: H_{S^{1}}^{*}(M ; \mathbb{Z}) \longrightarrow H^{*}\left(\mathbb{C P}^{\infty} ; \mathbb{Z}\right)$. Since this map is given by "integration over the fiber," we will usually denote it by the symbol $\int_{M}$. We will need the following theorem, due to Atiyah-Bott and Berline-Vergne [AB, BV]. 
Theorem 2.4. Let the circle act on a compact manifold $M$. Fix $\alpha \in H_{S^{1}}^{*}(M ; \mathbb{Q})$. As elements of $\mathbb{Q}(t)$,

$$
\int_{M} \alpha=\sum_{F \subset M^{S^{1}}} \int_{F} \frac{\left.\alpha\right|_{F}}{e_{S^{1}}\left(N_{F}\right)}
$$

where the sum is over all fixed components, and $e_{S^{1}}\left(N_{F}\right)$ denotes the equivariant Euler class of the normal bundle to $F$.

Remark 2.5. If $p \in M^{S^{1}}$ is an isolated fixed point, and the (integer) weights at $p$ are $\xi_{1}, \ldots, \xi_{n}$ (repeated with multiplicity), then $\left.c_{i}^{S^{1}}(M)\right|_{p}=\sigma_{i}\left(\xi_{1}, \ldots, \xi_{n}\right) t^{i}$, where $\sigma_{i}$ is the $i$ th elementary symmetric polynomial and $t$ is the generator of $H_{S^{1}}^{2}(p ; \mathbb{Z})$. For example, $c_{1}^{S^{1}}(M)=\sum \xi_{i} t$ and $e_{S^{1}}\left(N_{p}\right)=\left.c_{n}^{S^{1}}(M)\right|_{p}=\left(\prod \xi_{j}\right) t^{n}$. Hence,

$$
\int_{p} \frac{\left.c_{i}^{S^{1}}\right|_{p}}{e_{S^{1}}\left(N_{p}\right)}=\frac{\sigma_{i}\left(\xi_{1}, \ldots, \xi_{n}\right)}{\prod \xi_{j}} t^{i-n}
$$

If $\Sigma$ is a fixed surface of genus $g_{\Sigma}$ instead, then - since every vector bundle over a surface splits - the normal bundle to $\Sigma$ is the direct sum of line bundles with equivariant Chern classes $\xi_{1} t+a_{1} u, \ldots, \xi_{n-1} t+a_{n-1} u$, where $\xi_{1}, \ldots, \xi_{n-1}$ are the non-zero weights at $\Sigma$ (repeated with multiplicity), $u$ is the positive generator of $H^{2}(\Sigma ; \mathbb{Z})$, and the $a_{i}$ 's are integers. Since $e_{S^{1}}\left(N_{\Sigma}\right)=\left(\prod_{j} \xi_{j} t\right)\left(1+\frac{u}{t} \sum_{j} \frac{a_{j}}{\xi_{j}}\right)$ and $\left.1\right|_{\Sigma}=1$,

$$
\int_{\Sigma} \frac{\left.1\right|_{\Sigma}}{e_{S^{1}}\left(N_{\Sigma}\right)}=\int_{\Sigma} \frac{1}{\left(\prod_{j} \xi_{j} t\right)\left(1+\frac{u}{t} \sum_{j} \frac{a_{j}}{\xi_{j}}\right)}=\frac{\int_{\Sigma}\left(1-\frac{u}{t} \sum_{j} \frac{a_{j}}{\xi_{j}}\right)}{\prod_{j} \xi_{j} t}=-\frac{\sum_{j} \frac{a_{j}}{\xi_{j}}}{\prod_{j} \xi_{j}} t^{-n} .
$$

Similarly, since $\left.c_{1}^{S^{1}}(M)\right|_{\Sigma}=c_{1}(\Sigma)+u \sum_{j} a_{j}+t \sum_{j} \xi_{j}$,

$$
\begin{aligned}
\int_{\Sigma} \frac{\left.c_{1}^{S^{1}}(M)\right|_{\Sigma}}{e_{S^{1}}\left(N_{\Sigma}\right)} & =\frac{\int_{\Sigma}\left(c_{1}(\Sigma)+u \sum_{j} a_{j}+t \sum_{j} \xi_{j}\right)\left(1-\frac{u}{t} \sum_{j} \frac{a_{j}}{\xi_{j}}\right)}{\prod_{j} \xi_{j} t} \\
& =\frac{\int_{\Sigma}\left(c_{1}(\Sigma)-u \sum_{i \neq j} \frac{a_{j} \xi_{i}}{\xi_{j}}+t \sum_{j} \xi_{j}\right)}{\prod_{j} \xi_{j} t} \\
& =\frac{2\left(1-g_{\Sigma}\right)-\sum_{i \neq j} \frac{a_{j} \xi_{i}}{\xi_{j}}}{\prod_{j} \xi_{j}} t^{1-n} .
\end{aligned}
$$

Finally, since $\left(c_{1}^{S^{1}}(M)\right)^{2}-2 c_{2}^{S^{1}}(M)=t^{2} \sum_{j} \xi_{j}^{2}+2 u t \sum_{j} \xi_{j} a_{j}$,

$$
\begin{aligned}
\int_{\Sigma} \frac{\left.\left(c_{1}^{S^{1}}(M)\right)^{2}\right|_{\Sigma}-\left.2 c_{2}^{S^{1}}(M)\right|_{\Sigma}}{e_{S^{1}}\left(N_{\Sigma}\right)} & =\frac{\int_{\Sigma}\left(t^{2} \sum_{j} \xi_{j}^{2}+2 u t \sum_{j} \xi_{j} a_{j}\right)\left(1-\frac{u}{t} \sum_{j} \frac{a_{j}}{\xi_{j}}\right)}{\prod_{j} \xi_{j} t} \\
& =\frac{\int_{\Sigma}\left(u t \sum_{j} a_{j} \xi_{j}-u t \sum_{i \neq j} \frac{a_{j} \xi_{i}^{2}}{\xi_{j}}+t^{2} \sum_{j} \xi_{j}^{2}\right)}{\prod_{j} \xi_{j} t} \\
& =\frac{\sum_{j} a_{j} \xi_{j}-\sum_{i \neq j} \frac{a_{j} \xi_{i}^{2}}{\xi_{j}}}{\prod_{j} \xi_{j}} t^{2-n}
\end{aligned}
$$

Finally, we will need the following very simple lemmas. 
Lemma 2.6. Let the circle act on a compact symplectic manifold $(M, \omega)$. Let $p$ and $p^{\prime}$ be fixed points which lie in the same component $N$ of $M^{\mathbb{Z}_{k}}$, for some $k>1$. Then the $S^{1}$-weights at $p$ and at $p^{\prime}$ are equal modulo $k$.

Proof. Since $\mathbb{Z}_{k}$ fixes $N$, the weights of the representation of $\mathbb{Z}_{k}$ on the tangent space $T_{q} M$ are the same for all $q \in N$. Moreover, if $q \in N$ is fixed by the circle action, then the weights for the $\mathbb{Z}_{k}$-action on $T_{q} M$ are exactly the reduction modulo $k$ of the weights for the circle action.

Lemma 2.7. Let the circle act on a compact symplectic manifold $(M, \omega)$ with moment map $\Phi: M \longrightarrow \mathbb{R}$. There exists an equivariant extension $w \in H_{S^{1}}^{2}(M ; \mathbb{R})$ of $[\omega] \in H^{2}(M ; \mathbb{R})$ so that $\left.w\right|_{F^{\prime}}=\left[\left.\omega\right|_{F^{\prime}}\right]-\Phi\left(F^{\prime}\right) t$ for all fixed components $F^{\prime}$.

Proof. Take $w=[\omega-\Phi t]$ in the Borel model for equivariant cohomology.

Example 2.8. Let the torus $\left(S^{1}\right)^{3} \subset \mathrm{SU}(4)$ act on $\mathbb{C P}^{3}$ by

$$
\left(\lambda_{1}, \lambda_{2}, \lambda_{3}\right) \cdot\left[x_{0}, x_{1}, x_{2}, x_{3}\right]=\left[x_{0}, \lambda_{1} x_{1}, \lambda_{2} x_{2}, \lambda_{3} x_{3}\right] .
$$

Let $e_{1}, e_{2}$ and $e_{3}$ denote the standard basis for the weight lattice $\left(\mathbb{Z}^{3}\right)^{*}$. The fixed points are $[1,0,0,0],[0,1,0,0],[0,0,1,0]$, and $[0,0,0,1]$; the weights at these points are $\left\{e_{1}, e_{2}, e_{3}\right\},\left\{-e_{1}, e_{2}-e_{1}, e_{3}-e_{1}\right\},\left\{-e_{2}, e_{1}-e_{2}, e_{3}-e_{2}\right\}$, and $\left\{-e_{3}, e_{1}-e_{3}\right.$, $\left.e_{2}-e_{3}\right\}$, respectively.

Example 2.9. Let the torus $\left(S^{1}\right)^{2} \subset \mathrm{SO}(5)$ act on $\widetilde{G}_{2}\left(\mathbb{R}^{5}\right)$ induced by the $S^{1}$ action on $\mathbb{R}^{5}=\mathbb{R} \times \mathbb{C}^{2}$ given by

$$
\left(\lambda_{1}, \lambda_{2}\right) \cdot\left(t, x_{1}, x_{2}\right)=\left(t, \lambda_{1} x_{1}, \lambda_{2} x_{2}\right) .
$$

Let $e_{1}$ and $e_{2}$ denote the standard basis for the weight lattice $\left(\mathbb{Z}^{2}\right)^{*}$. The fixed points are the planes $\left\{\left(y_{1}, \ldots, y_{5}\right) \in \mathbb{R}^{5} \mid y_{1}=y_{4}=y_{5}=0\right\}$ and $\left\{\left(y_{1}, \ldots, y_{5}\right) \in\right.$ $\left.\mathbb{R}^{5} \mid y_{1}=y_{2}=y_{3}=0\right\}$, with either orientation; the weights at these points are $\left\{e_{1}, e_{1}+e_{2}, e_{1}-e_{2}\right\},\left\{-e_{1},-e_{1}+e_{2},-e_{1}-e_{2}\right\},\left\{e_{2}, e_{2}+e_{1}, e_{2}-e_{1}\right\}$, and $\left\{-e_{2},-e_{2}+e_{1},-e_{2}-e_{1}\right\}$.

Remark 2.10. The cases (C) and (D) described in Theorem 1 are consistent with Wu's theorem, so we cannot use this theorem to rule out such manifolds. Wu's theorem states that the total Steifel-Whitney class $w(M)$ of a connected manifold $M$ is equal to $\mathrm{Sq}(v(M))$, where $\mathrm{Sq}: H^{*}\left(M ; \mathbb{Z}_{2}\right) \longrightarrow H^{*}\left(M ; \mathbb{Z}_{2}\right)$ is the Steenrod square operator and $v(M) \in H^{*}\left(M ; \mathbb{Z}_{2}\right)$ is the unique class so that $v(M) \cup x=$ $\mathrm{Sq}(x)$ for all $x \in H^{*}\left(M ; \mathbb{Z}_{2}\right)$. (See $\S 18.8$ in $[\mathrm{Hu}]$.) For example, if $H^{*}\left(M ; \mathbb{Z}_{2}\right)=$ $\mathbb{Z}_{2}[x] /\left(x^{4}\right)$, as in case $(\mathrm{C})$, then $\mathrm{Sq}^{2}\left(x^{2}\right)=0$ and so Wu's theorem implies that $w(M)=0$. Since $w(M)$ is the image of $c(M)$ under the coefficient homomorphism $H^{*}(M ; \mathbb{Z}) \longrightarrow H^{*}\left(M ; \mathbb{Z}_{2}\right)$, this is satisfied in both cases. Similarly, if $H^{*}\left(M ; \mathbb{Z}_{2}\right)=$ $\mathbb{Z}_{2}[x, y] /\left(x^{2}, y^{2}\right)$, as in case (D), then either $\operatorname{Sq}^{2}(y)=0$ and $w(M)=1$, or $\mathrm{Sq}^{2}(y)=$ $x y$ and $w(M)=1+x$. The latter statement is satisfied in both cases.

Remark 2.11. Similarly, cases (C) and (D) are consistent with the fixed point formula for the Hirzebruch genus. Let $M$ be a compact almost complex manifold; let $\chi_{y}$ denote the Hirzebruch genus corresponding to the power series

$$
\frac{x\left(1+y e^{-x(1+y)}\right)}{1-e^{-x(1+y)}} .
$$


On the one hand, if $M$ is 6-dimensional, then a direct calculation shows that

$$
\chi_{y}(M)=\frac{1}{24}\left(1+y-y^{2}-y^{3}\right) \int_{M} c_{1}(M) c_{2}(M)+\frac{1}{2}\left(-y+y^{2}\right) \int_{M} c_{1}(M) .
$$

On the other hand, if a circle acts on $M$, then by [HBJ], $\chi_{y}(M)=\sum_{F}(-y)^{\lambda_{F}} \chi_{y}(F)$, where the sum is over all fixed components. In particular, in cases (C) and (D),

$$
\chi_{y}(M)=1-y+y^{2}-y^{3} .
$$

Since $\int_{M} c_{1}(M) c_{2}(M)=24$ and $\int_{M} c_{3}(M)=4$ in both cases, these formulas agree.

\section{Arbitrary dimensions}

We begin by exploring some of the consequences of our central assumptions in arbitrary dimensions. More precisely, let a circle act in a Hamiltonian fashion on a compact symplectic manifold $(M, \omega)$; assume that $H^{i}(M ; \mathbb{Z})=H^{i}\left(\mathbb{C} \mathbb{P}^{n} ; \mathbb{Z}\right)$ for all $i$. Our main result is that the fixed point data determines the (equivariant) cohomology ring and Chern classes; see Proposition 3.9. We also show, in Proposition [3.4, that the index of the fixed components determines the order of their moment images.

Roughly speaking, our first result states that critical components near the minimum must have low index.

Lemma 3.1. Let the circle act on a compact symplectic manifold $(M, \omega)$ with moment map $\Phi: M \longrightarrow \mathbb{R}$. Given any fixed component $F$,

$$
\lambda_{F} \leq \sum_{\Phi\left(F^{\prime}\right)<\Phi(F)}\left(\frac{1}{2} \operatorname{dim}\left(F^{\prime}\right)+1\right),
$$

where the sum is over all fixed components $F^{\prime}$ such that $\Phi\left(F^{\prime}\right)<\Phi(F)$.

Proof. Pick a fixed component $F$. Let $N=\sum_{\Phi\left(F^{\prime}\right)<\Phi(F)}\left(\frac{1}{2} \operatorname{dim}\left(F^{\prime}\right)+1\right)$. By Lemma 2.7. there exists an equivariant extension $w \in H_{S^{1}}^{2}(M ; \mathbb{R})$ of $\omega$ so that $\left.w\right|_{F^{\prime}}=\left[\left.\omega\right|_{F^{\prime}}\right]-\Phi\left(F^{\prime}\right) t$ for all fixed components $F^{\prime}$. Define $\beta \in H_{S^{1}}^{2 N}(M ; \mathbb{R})$ by

$$
\beta=\prod_{\Phi\left(F^{\prime}\right)<\Phi(F)}\left(w+\Phi\left(F^{\prime}\right) t\right)^{\frac{1}{2} \operatorname{dim}\left(F^{\prime}\right)+1},
$$

where now the product is over all fixed components $F^{\prime}$ such that $\Phi\left(F^{\prime}\right)<\Phi(F)$. Given any fixed component $F^{\prime}$,

$$
\left(\left.\left(w+\Phi\left(F^{\prime}\right) t\right)\right|_{F^{\prime}}\right)^{\frac{1}{2} \operatorname{dim}\left(F^{\prime}\right)+1}=\left[\left.\omega\right|_{F^{\prime}}\right]^{\frac{1}{2} \operatorname{dim}\left(F^{\prime}\right)+1}=0 .
$$

Hence, the restriction $\left.\beta\right|_{F^{\prime}}$ vanishes for all fixed components $F^{\prime}$ such that $\Phi\left(F^{\prime}\right)<$ $\Phi(F)$. In contrast, as a polynomial in $t$,

$$
\begin{aligned}
\left.\beta\right|_{F} & =\prod_{\Phi\left(F^{\prime}\right)<\Phi(F)}\left(\left.\left(w+\Phi\left(F^{\prime}\right) t\right)\right|_{F}\right)^{\frac{1}{2} \operatorname{dim}\left(F^{\prime}\right)+1} \\
& =\prod_{\Phi\left(F^{\prime}\right)<\Phi(F)}\left(\left[\left.\omega\right|_{F}\right]+\Phi(F) t-\Phi\left(F^{\prime}\right) t\right)^{\frac{1}{2} \operatorname{dim}\left(F^{\prime}\right)+1} \\
& =\prod_{\Phi\left(F^{\prime}\right)<\Phi(F)}\left(\Phi(F) t-\Phi\left(F^{\prime}\right) t\right)^{\frac{1}{2} \operatorname{dim}\left(F^{\prime}\right)+1}+\text { lower order terms. }
\end{aligned}
$$

Hence $\left.\beta\right|_{F} \neq 0$. Since $\left.\beta\right|_{F}$ is a multiple of $e_{S^{1}}\left(N_{F}^{-}\right)$by Corollary 2.3. this implies that $N \geq \lambda_{F}$. 
Remark 3.2. In fact, by eliminating some of the redundant factors of $\beta$, one can show that there exist fixed components $F_{1}, \ldots, F_{k}$ such that $\Phi\left(F_{i}\right)<\Phi(F)$ for all $i, \lambda_{F} \leq \sum_{i}\left(\frac{1}{2} \operatorname{dim}\left(F_{i}\right)+1\right)$, and $\Phi\left(F_{i}\right) \neq \Phi\left(F_{j}\right)$ for all $i \neq j$.

When $H^{2 i}(M ; \mathbb{R})=H^{2 i}\left(\mathbb{C P}^{n} ; \mathbb{R}\right)$ for all $i$, there are not many fixed components, and so this determines the order of the fixed components under the moment map.

Lemma 3.3. Let the circle act on a compact symplectic manifold $(M, \omega)$ with moment map $\Phi: M \longrightarrow \mathbb{R} ;$ assume that $H^{2 i}(M ; \mathbb{R})=H^{2 i}\left(\mathbb{C P}^{n} ; \mathbb{R}\right)$ for all $i$. There exists a unique fixed component $F$ such that $2 \lambda_{F} \leq 2 i \leq 2 \lambda_{F}+\operatorname{dim}(F)$ for all $i$ such that $0 \leq 2 i \leq 2 n$.

Proof. Since every fixed component $F$ is symplectic, $H^{2 i}(F ; \mathbb{R}) \neq 0$ for every integer $i$ such that $0 \leq 2 i \leq \operatorname{dim}(F)$. The first claim thus follows immediately from the assumption and the fact that moment maps are perfect Morse-Bott functions.

Proposition 3.4. Let the circle act on a compact symplectic manifold $(M, \omega)$ with moment map $\Phi: M \longrightarrow \mathbb{R} ;$ assume that $H^{2 i}(M ; \mathbb{R})=H^{2 i}\left(\mathbb{C P}^{n} ; \mathbb{R}\right)$ for all $i$. Then for all fixed components $F$ and $F^{\prime}$,

$$
\Phi\left(F^{\prime}\right)<\Phi(F) \quad \text { exactly if } \quad \lambda_{F^{\prime}}<\lambda_{F} .
$$

Proof. Consider any fixed component $F$. Define

$$
N=\sum_{\Phi\left(F^{\prime}\right)<\Phi(F)}\left(\frac{1}{2} \operatorname{dim}\left(F^{\prime}\right)+1\right)
$$

where the sum is over all fixed components $F^{\prime}$ such that $\Phi\left(F^{\prime}\right)<\Phi(F)$. Since $H^{2 i}(M ; \mathbb{R})=H^{2 i}\left(\mathbb{C P}^{n} ; \mathbb{R}\right)$ for all $i$, Lemma 3.3 implies that there exists a unique fixed component $F^{\prime}$ such that $2 \lambda_{F^{\prime}} \leq 2 i \leq 2 \lambda_{F^{\prime}}+\operatorname{dim}\left(F^{\prime}\right)$ for all $i$ such that $0 \leq 2 i \leq 2 n$. Therefore, since the fixed components are all even-dimensional,

$$
\sum_{\lambda_{F^{\prime}}<N}\left(\frac{1}{2} \operatorname{dim}\left(F^{\prime}\right)+1\right) \leq N,
$$

where here the sum is over all fixed components $F^{\prime}$ such that $\lambda_{F^{\prime}}<N$. On the other hand, by Lemma 3.1, for any fixed component $F^{\prime}$ with $\Phi\left(F^{\prime}\right) \leq \Phi(F)$,

$$
\lambda_{F^{\prime}} \leq \sum_{\Phi\left(F^{\prime \prime}\right)<\Phi\left(F^{\prime}\right)}\left(\frac{1}{2} \operatorname{dim}\left(F^{\prime \prime}\right)+1\right) \leq \sum_{\Phi\left(F^{\prime \prime}\right)<\Phi(F)}\left(\frac{1}{2} \operatorname{dim}\left(F^{\prime \prime}\right)+1\right)=N,
$$

with equality impossible unless $\Phi\left(F^{\prime}\right)=\Phi(F)$. In particular, $\lambda_{F^{\prime}}<N$ for all fixed components $F^{\prime}$ such that $\Phi\left(F^{\prime}\right)<\Phi(F)$, and so equations (3.5) and (3.6) imply that $\lambda_{F^{\prime}}<N$ exactly if $\Phi\left(F^{\prime}\right)<\Phi(F)$. Since $\lambda_{F} \leq N$, we can conclude that $\lambda_{F}=N$; this proves the claim.

For our main proposition, we will also need the fact that the moment map is also a perfect Morse-Bott function over any field whenever $H^{2 i}(M ; \mathbb{R})=H^{2 i}\left(\mathbb{C P}^{n} ; \mathbb{R}\right)$ for all $i$.

Lemma 3.7. Let the circle act on a compact symplectic manifold $(M, \omega)$ with moment map $\Phi: M \longrightarrow \mathbb{R} ;$ assume that $H^{2 i}(M ; \mathbb{R})=H^{2 i}\left(\mathbb{C P}^{n} ; \mathbb{R}\right)$ for all $i$. Then

$$
H^{j}(M ; \mathbb{Z})=\bigoplus_{F \subset M^{S^{1}}} H^{j-2 \lambda_{F}}(F ; \mathbb{Z}) \quad \forall j,
$$

where the sum is over all fixed components. 
Proof. Consider a fixed component $F$ and integer $j$ so that $H^{j-2 \lambda_{F}}(F ; \mathbb{Z}) \neq 0$. Let $M^{ \pm}=\Phi^{-1}(-\infty, \Phi(F) \pm \epsilon)$ for $\epsilon>0$ sufficiently small. Since each fixed component is even-dimensional, Lemma 3.3 implies that $H^{j-2 \lambda_{F^{\prime}}}\left(F^{\prime} ; \mathbb{Z}\right)=H^{j-2 \lambda_{F^{\prime}}+1}\left(F^{\prime} ; \mathbb{Z}\right)=0$ for all fixed components $F^{\prime} \neq F$. Therefore, $H^{j}\left(M^{-} ; \mathbb{Z}\right)=H^{j+1}\left(M^{-} ; \mathbb{Z}\right)=0$, and so $H^{j}\left(M^{+} ; \mathbb{Z}\right)=H^{j-2 \lambda_{F}}(F ; \mathbb{Z})$. By a similar argument, $H^{j}\left(M^{+} ; \mathbb{Z}\right)=H^{j}(M ; \mathbb{Z})$.

Given any fixed component $F$, let $\Gamma_{F} \in \mathbb{Z}$ denote the sum of the weights at $F$.

Lemma 3.8. Let the circle act on a compact symplectic manifold $(M, \omega)$ with moment map $\Phi: M \longrightarrow \mathbb{R}$. Then $c_{1}(M) \neq 0$.

Proof. Given a point $p$ in a fixed component $F,\left.c_{1}^{S^{1}}(M)\right|_{p}=\Gamma_{F} t$. Each weight at the minimal fixed component $F$ is positive, while each weight at the maximal fixed component $F^{\prime}$ is negative. Hence, $\Gamma_{F} \neq \Gamma_{F^{\prime}}$; this implies that $c_{1}^{S^{1}}(M)$ is not a multiple of $t$.

Finally, we state our main proposition.

Proposition 3.9. Let the circle act on a compact symplectic manifold $(M, \omega)$ with moment map $\Phi: M \longrightarrow \mathbb{R} ;$ assume that $H^{j}(M ; \mathbb{Z})=H^{j}\left(\mathbb{C P}^{n} ; \mathbb{Z}\right)$ for all $j$.

For each integer $i$ such that $0 \leq 2 i \leq 2 n$, there exists a unique fixed component $F_{i}$ so that $H^{2 i-2 \lambda_{F_{i}}}\left(F_{i} ; \mathbb{Z}\right)=\mathbb{Z}$; let $u_{i}$ be a generator of $H^{2 i-2 \lambda_{F_{i}}}\left(F_{i} ; \mathbb{Z}\right)$, and let $\left.c_{1}(M)^{i-\lambda_{F_{i}}}\right|_{F_{i}}=m_{i} u_{i}$. Then the cohomology class

$$
\alpha_{i}=\frac{\Lambda_{F_{i}}^{-}}{m_{i}}\left(c_{1}^{S^{1}}(M)-\Gamma_{F_{i}} t\right)^{i-\lambda_{F_{i}}} \prod_{\lambda_{F^{\prime}}<\lambda_{F_{i}}}\left(\frac{c_{1}^{S^{1}}(M)-\Gamma_{F^{\prime}} t}{\Gamma_{F_{i}}-\Gamma_{F^{\prime}}}\right)^{\frac{1}{2} \operatorname{dim}\left(F^{\prime}\right)+1}
$$

is well-defined and lies in $H_{S^{1}}^{2 i}(M ; \mathbb{Z})$. (Here, the product is over all fixed components $F^{\prime}$ such that $\lambda_{F^{\prime}}<\lambda_{F_{i}}$.) Moreover, the classes $\alpha_{0}, \ldots, \alpha_{n}$ form a basis for $H_{S^{1}}^{*}(M ; \mathbb{Z})$ as an $H^{*}\left(\mathbb{C P} \mathbb{P}^{\infty} ; \mathbb{Z}\right)=\mathbb{Z}[t]$ module.

Proof. By Lemma 3.3, for each integer $i$ such that $0 \leq 2 i \leq 2 n$ there exists a unique fixed component $F_{i}$ such that $2 \lambda_{F_{i}} \leq 2 i \leq 2 \lambda_{F_{i}}+\operatorname{dim}\left(F_{i}\right)$. By Lemma 3.7 . $H^{2 i-2 \lambda_{F_{i}}}\left(F_{i} ; \mathbb{Z}\right) \simeq \mathbb{Z}$; let $u_{i}$ be a generator. Then $u_{0}, \ldots, u_{n}$ is a basis for $H^{*}\left(M^{S^{1}}\right)$. By Proposition 2.2, for each such $i$ there exists a class $\alpha_{i} \in H_{S^{1}}^{2 i}(M ; \mathbb{Z})$ so that

(1) $\left.\alpha_{i}\right|_{F_{i}}=u_{i} e_{S^{1}}\left(N_{F_{i}}^{-}\right)$, and

(2) $\left.\alpha_{i}\right|_{F^{\prime}}=0$ for all other fixed components $F^{\prime}$ with $\Phi\left(F^{\prime}\right) \leq \Phi\left(F_{i}\right)$.

Moreover, $\alpha_{0}, \ldots, \alpha_{n}$ is a basis for $H_{S^{1}}^{*}(M ; \mathbb{Z})$ as an $H^{*}\left(\mathbb{C P}^{\infty} ; \mathbb{Z}\right)$ module.

Since each fixed component has even dimension, Lemma 3.3 implies that for each fixed component $F$,

$$
\sum_{\lambda_{F^{\prime}}<\lambda_{F}}\left(\frac{1}{2} \operatorname{dim}\left(F^{\prime}\right)+1\right)=\lambda_{F}
$$

where here the sum is over all fixed components $F^{\prime}$ such that $\lambda_{F^{\prime}}<\lambda_{F}$. Hence, for each $i$ such that $0 \leq 2 i \leq \operatorname{dim}(M)$ we may define

$$
\beta_{i}=\left(c_{1}^{S^{1}}(M)-\Gamma_{F_{i}} t\right)^{i-\lambda_{F_{i}}} \prod_{\lambda_{F^{\prime}}<\lambda_{F_{i}}}\left(c_{1}^{S^{1}}(M)-\Gamma_{F^{\prime}} t\right)^{\frac{1}{2} \operatorname{dim}\left(F^{\prime}\right)+1} \in H_{S^{1}}^{2 i}(M ; \mathbb{Z}) .
$$

Moreover, for every fixed component $F^{\prime}$,

$$
\left.\left(c_{1}^{S^{1}}(M)-\Gamma_{F^{\prime}} t\right)^{\frac{1}{2} \operatorname{dim}\left(F^{\prime}\right)+1}\right|_{F^{\prime}}=\left(\left.c_{1}(M)\right|_{F^{\prime}}\right)^{\frac{1}{2} \operatorname{dim}\left(F^{\prime}\right)+1}=0 .
$$


Therefore, the restriction $\left.\beta_{i}\right|_{F^{\prime}}$ vanishes for every fixed component $F^{\prime}$ such that $\lambda_{F^{\prime}}<\lambda_{F_{i}}$. Combining Corollary 2.3 and Proposition 3.4, this implies that

$$
\beta_{i}=\sum_{\lambda_{F_{j}} \geq \lambda_{F_{i}}} x_{j} \alpha_{j}
$$

where $x_{j} \in H^{*}\left(\mathbb{C P}^{\infty} ; \mathbb{Z}\right)=\mathbb{Z}[t]$ for all $j$. Here, the sum is over $j$ such that $\lambda_{F_{j}} \geq \lambda_{F_{i}}$. Since $\beta_{i} \in H_{S^{1}}^{2 i}(M ; \mathbb{Z})$, Lemma 3.3 and degree considerations imply that

$$
\beta_{i}=\sum_{\substack{F_{j}=F_{i} \\ j \leq i}} x_{j} \alpha_{j},
$$

where now the sum is over $j$ such that $F_{j}=F_{i}$ and $j \leq i$.

Additionally, recall that $\left.c_{1}(M)^{i-\lambda_{F_{i}}}\right|_{F_{i}}=m_{i} u_{i}$. As a polynomial in $t$,

$$
\begin{aligned}
\left.\beta_{i}\right|_{F_{i}} & =\left.c_{1}(M)^{i-\lambda_{F_{i}}}\right|_{F_{i}} \prod_{\lambda_{F^{\prime}}<\lambda_{F}}\left(\left.c_{1}(M)\right|_{F}+\left(\Gamma_{F}-\Gamma_{F^{\prime}}\right) t\right)^{\frac{1}{2} \operatorname{dim}\left(F^{\prime}\right)+1} \\
& =m_{i} u_{i} \prod_{\lambda_{F^{\prime}}<\lambda_{F_{i}}}\left(\Gamma_{F_{i}}-\Gamma_{F^{\prime}}\right)^{\frac{1}{2} \operatorname{dim}\left(F^{\prime}\right)+1} t^{\lambda_{F_{i}}}+\text { lower order terms. }
\end{aligned}
$$

On the other hand, for any $j$ such that $F_{j}=F_{i}$,

$$
\left.\alpha_{j}\right|_{F_{i}}=u_{j} e_{S^{1}}\left(N_{F_{i}}^{-}\right)=u_{j} \Lambda_{F_{i}}^{-} t^{\lambda_{F_{i}}}+\text { lower order terms. }
$$

Comparing equations (3.10), (3.11), and (3.12), we see that

$$
\beta_{i}=\frac{m_{i}}{\Lambda_{F_{i}}^{-}} \prod_{\lambda_{F^{\prime}}<\lambda_{F_{i}}}\left(\Gamma_{F_{i}}-\Gamma_{F^{\prime}}\right)^{\frac{1}{2} \operatorname{dim}\left(F^{\prime}\right)+1} \alpha_{i} .
$$

Finally, by Lemma 3.8, $c_{1}(M) \neq 0$. Since $H^{2}(M ; \mathbb{R})=\mathbb{R}$, this implies that $c_{1}(M)$ is a non-zero multiple of $\omega$. Therefore, $c_{1}(M)^{i} \neq 0$. Since $\beta_{i}$ maps to $c_{1}(M)^{i}$ under the natural restriction map from the equivariant cohomology of $M$ to the ordinary cohomology, this clearly implies that $\beta_{i} \neq 0$.

We can use this proposition (and Remark 2.4) to obtain a particularly nice description of the equivariant cohomology and total Chern class in the following special cases.

Corollary 3.13. Let the circle act on compact symplectic manifolds $(M, \omega)$ and $(\widehat{M}, \widehat{\omega})$ with moment maps $\Phi: M \longrightarrow \mathbb{R}$ and $\widehat{\Phi}: \widehat{M} \longrightarrow \mathbb{R}$, respectively; assume that $H^{j}(\widehat{M} ; \mathbb{Z})=H^{j}\left(\mathbb{C P} \mathbb{P}^{n} ; \mathbb{Z}\right)$ for all $j$. If $f: M^{S^{1}} \longrightarrow \widehat{M}^{S^{1}}$ is a diffeomorphism and $\left.T(M)\right|_{M^{S^{1}}} \cong f^{*}\left(\left.T(\widehat{M})\right|_{\widehat{M}^{S^{1}}}\right)$, then $\left.H_{S^{1}}^{*}(M ; \mathbb{Z})\right|_{M^{S^{1}}}=f^{*}\left(\left.H_{S^{1}}^{*}(\widehat{M} ; \mathbb{Z})\right|_{\widehat{M}^{S^{1}}}\right)$ and $\left.c(M)\right|_{M^{S^{1}}}=f^{*}\left(\left.c(\widehat{M})\right|_{\widehat{M}^{S^{1}}}\right)$.

Proof. Lemma 3.7 implies that

$$
\bigoplus_{\widehat{F} \subset \widehat{M}^{S^{1}}} H^{j-2 \lambda_{\widehat{F}}}(\widehat{F} ; \mathbb{Z})=H^{j}(\widehat{M} ; \mathbb{Z})=H^{j}\left(\mathbb{C P}^{n} ; \mathbb{Z}\right) \quad \forall j .
$$

Since, $\left.T(M)\right|_{M^{S^{1}}} \cong f^{*}\left(\left.T(\widehat{M})\right|_{\widehat{M}^{S^{1}}}\right)$, this implies that

$$
\bigoplus_{F \subset M^{S^{1}}} H^{j-2 \lambda_{F}}(F ; \mathbb{Z})=H^{j}\left(\mathbb{C P}^{n} ; \mathbb{Z}\right) \quad \forall j .
$$


As in Lemma 3.7, this implies that

$$
H^{j}(M ; \mathbb{Z})=H^{j}\left(\mathbb{C} \mathbb{P}^{n} ; \mathbb{Z}\right) \quad \forall j .
$$

The claim now follows immediately from Proposition 3.9

Corollary 3.14. Let the circle act on a compact symplectic manifold $(M, \omega)$ with moment map $\Phi: M \longrightarrow \mathfrak{t}^{*}$; assume that there is a unique fixed point of index $2 i$ for all $i$ such that $0 \leq i \leq n$. As an $H^{*}\left(\mathbb{C P}^{\infty} ; \mathbb{Z}\right)=\mathbb{Z}[t]$ module, $H_{S^{1}}^{*}(M ; \mathbb{Z})$ is freely generated by $1, \alpha_{1}, \ldots, \alpha_{n}$, where

$$
\alpha_{i}=\Lambda_{p_{i}}^{-} \prod_{j=0}^{i-1} \frac{c_{1}^{S^{1}}(M)-\Gamma_{p_{j}} t}{\Gamma_{p_{i}}-\Gamma_{p_{j}}} .
$$

(In particular, $\alpha_{i} \in H_{S^{1}}^{2 i}(M ; \mathbb{Z})$ for all $i$.)

Example 3.15. Let the circle act on a compact symplectic manifold $(M, \omega)$ with moment map $\Phi: M \longrightarrow \mathfrak{t}^{*}$. Assume that the fixed set consists of four points $p_{0}, p_{1}, p_{2}$ and $p_{3}$ with weights $\{1,2,3\},\{1,-1, l\},\{1,-1,-l\}$, and $\{-1,-2,-3\}$, respectively. As an $H^{*}\left(\mathbb{C P}^{\infty} ; \mathbb{Z}\right)=\mathbb{Z}[t]$ module, $H_{S^{1}}^{*}(M ; \mathbb{Z})$ is freely generated by $1, \alpha_{1}, \alpha_{2}, \alpha_{3}$, where

$$
\begin{gathered}
\left.\alpha_{1}\right|_{p_{1}}=t,\left.\alpha_{1}\right|_{p_{2}}=\frac{6+l}{6-l} t,\left.\alpha_{1}\right|_{p_{3}}=\frac{12}{6-l} t,\left.\alpha_{2}\right|_{p_{2}}=l t^{2}, \\
\left.\alpha_{2}\right|_{p_{3}}=6 t^{2},\left.\alpha_{3}\right|_{p_{3}}=6 t^{3}, \text { and }\left.\alpha_{i}\right|_{p_{j}}=0 \forall j<i \text {; moreover, } \\
\left.c^{S^{1}}(M)\right|_{p_{0}}=1+6 t+11 t^{2}+6 t^{3},\left.c^{S^{1}}(M)\right|_{p_{1}}=1+l t-t^{2}-l t^{3}, \\
\left.c^{S^{1}}(M)\right|_{p_{2}}=1-l t-t^{2}+l t^{3}, \text { and }\left.c^{S^{1}}(M)\right|_{p_{3}}=1-6 t+11 t^{2}-6 t^{3} .
\end{gathered}
$$

In the above cases, the fixed set is torsion-free and so formality holds. Thus, the ordinary cohomology $H^{*}(M ; \mathbb{Z})$ and total Chern class $c(M)$ are also very easy to describe.

Corollary 3.16. Let the circle act on compact symplectic manifolds $(M, \omega)$ and $(\widehat{M}, \widehat{\omega})$ with moment maps $\Phi: M \longrightarrow \mathbb{R}$ and $\widehat{\Phi}: \widehat{M} \longrightarrow \mathbb{R}$, respectively; assume that $H^{j}(\widehat{M} ; \mathbb{Z})=H^{j}\left(\mathbb{C} \mathbb{P}^{n} ; \mathbb{Z}\right)$ for all $j$. If $f: M^{S^{1}} \longrightarrow \widehat{M}^{S^{1}}$ is a diffeomorphism and $\left.T(M)\right|_{M^{S^{1}}} \cong f^{*}\left(\left.T(\widehat{M})\right|_{\widehat{M} S^{1}}\right)$, then $f$ induces an isomorphism $f^{\sharp}: H^{*}(\widehat{M} ; \mathbb{Z}) \longrightarrow$ $H^{*}(M ; \mathbb{Z})$ so that $c(M)=f^{\sharp}(c(\widehat{M}))$.

Remark 3.17. More generally, let the circle act on compact symplectic manifolds $(M, \omega)$ and $(\widehat{M}, \widehat{\omega})$ with moment maps $\Phi: M \longrightarrow \mathbb{R}$ and $\widehat{\Phi}: \widehat{M} \longrightarrow \mathbb{R}$, respectively; let $f: M^{S^{1}} \longrightarrow \widehat{M}^{S^{1}}$ be an orientation-preserving diffeomorphism such that $\left.T(M)\right|_{M^{S^{1}}} \cong f^{*}\left(\left.T(\widehat{M})\right|_{\widehat{M} S^{1}}\right)$.

Since moment maps are (equivariantly) perfect Morse-Bott functions, $M$ and $\widehat{M}$ have the same (equivariant) Betti numbers. Moreover, Theorem 2.4 immediately implies that $M$ and $\widehat{M}$ have the same (equivariant) Chern numbers. Alternately, by GGK, $M$ and $\widehat{M}$ are cobordant as oriented equivariant stable-complex manifolds, and so have the same (equivariant) Chern numbers. Additionally, if $H_{2}(M ; \mathbb{R})=$ $H_{2}(\widehat{M} ; \mathbb{R})$, then since $c_{1}(M) \neq 0$ by Lemma 3.8 $c_{1}^{S^{1}}(M)$ and $t$ are a basis for $H_{S^{1}}^{2}(M ; \mathbb{R})$ as a vector space. By assumption,

$$
f^{*}\left(\left.c^{S^{1}}(\widehat{M})\right|_{\widehat{M}^{S^{1}}}\right)=\left.c^{S^{1}}(M)\right|_{M^{S^{1}}} .
$$


Hence, after possibly multiplying $\widehat{\omega}$ by a constant and adding a constant to $\widehat{\Phi}$,

$$
f^{*}\left(\left.[\widehat{\omega}-\widehat{\Phi} t]\right|_{\widehat{M}^{1}}\right)=\left.[\omega-\Phi t]\right|_{M^{S^{1}}} .
$$

Therefore, by [GGK], $M$ and $\widehat{M}$ are also cobordant as Hamiltonian $S^{1}$-spaces. This (or Theorem (2.4) implies that $M$ and $\widehat{M}$ have the same Duistermaat-Heckman measure. More generally, any product of equivariant Chern classes and powers of the class $[\omega-\Phi t]$ have the same integral over $M$ and $\widehat{M}$.

However, in general, cobordant manifolds do not have isomorphic (equivariant) cohomology rings. For example, let $M$ be the blow-up of $\mathbb{C P}^{2}$ at $[0,1,0]$, where $S^{1}$ acts on $\mathbb{C P}^{2}$ by

$$
\lambda \cdot\left[x_{0}, x_{1}, x_{2}\right]=\left[x_{0}, \lambda x_{1}, \lambda^{2} x_{2}\right],
$$

and let $S^{1}$ act on $\widehat{M}=\mathbb{C P}^{1} \times \mathbb{C P}^{1}$ by

$$
\lambda \cdot\left(\left[y_{0}, y_{1}\right],\left[z_{0}, z_{1}\right]\right)=\left(\left[y_{0}, \lambda y_{1}\right],\left[z_{0}, \lambda^{2} z_{1}\right]\right) .
$$

For the appropriate choice of symplectic forms, $M$ and $\widehat{M}$ are cobordant as stablecomplex Hamiltonian $S^{1}$-spaces, but as rings $H^{*}(M ; \mathbb{Z}) \nsucceq H^{*}(\widehat{M} ; \mathbb{Z})$.

Remark 3.18. Alternately, let the circle act on compact symplectic manifolds $(M, \omega)$ and $(\widehat{M}, \widehat{\omega})$ with moment maps $\Phi: M \longrightarrow \mathbb{R}$ and $\widehat{\Phi}: \widehat{M} \longrightarrow \mathbb{R}$, respectively; assume that $H^{j}(\widehat{M} ; \mathbb{Z})=H^{j}\left(\mathbb{C P}^{n} ; \mathbb{Z}\right)$ for all $j$. Also assume that there is a bijection from the fixed components $F_{1}, \ldots, F_{k}$ of $M$ to the fixed components $\widehat{F}_{1}, \ldots, \widehat{F}_{k}$ of $\widehat{M}$ and that there exists an isomorphism $f^{*}: H_{S^{1}}^{*}\left(\widehat{F}_{i} ; \mathbb{Z}\right) \longrightarrow H_{S^{1}}^{*}\left(F_{i} ; \mathbb{Z}\right)$ such that $f^{*}\left(\left.c^{S^{1}}(\widehat{M})\right|_{\widehat{F}_{i}}\right)=\left.c^{S^{1}}(M)\right|_{F_{i}}$ for all $i$. Then all the symmetric polynomials in the weights at $F$ and $\widehat{F}$ are the same, and so they have the same weights, and hence the same index. Therefore, the conclusions of Corollaries 3.13 and 3.13 still hold.

Corollary 3.19. Let the circle act on a compact symplectic manifold $(M, \omega)$ with moment map $\Phi: M \longrightarrow \mathfrak{t}^{*}$; assume that there is a unique fixed point $p_{i}$ of index $2 i$ for all $i$ such that $0 \leq 2 i \leq 2 n$. As a group, $H^{*}(M ; \mathbb{Z})$ is freely generated by $1, \widetilde{\alpha}_{1}, \ldots, \widetilde{\alpha}_{n}$, where

$$
\begin{gathered}
\widetilde{\alpha}_{i}=\frac{\Lambda_{p_{i}}^{-}}{\left(\Lambda_{p_{1}}^{-}\right)^{i}} \frac{\left(\Gamma_{p_{1}}-\Gamma_{p_{0}}\right)^{i}}{\prod_{j=0}^{i-1}\left(\Gamma_{p_{i}}-\Gamma_{p_{j}}\right)}\left(\widetilde{\alpha}_{1}\right)^{i} ; \text { moreover, } \\
c_{i}(M)=\frac{\Lambda_{p_{i}}^{+}}{\prod_{j=i+1}^{n}\left(\Gamma_{p_{i}}-\Gamma_{p_{j}}\right)}\left(\left.\sum_{k=0}^{i} c_{i}^{S^{1}}(M)\right|_{p_{k}} \frac{\prod_{j=i+1}^{n}\left(\Gamma_{p_{k}}-\Gamma_{p_{j}}\right)}{t^{i} \Lambda_{p_{k}}}\right) \widetilde{\alpha}_{i} \\
=\frac{1}{\Lambda_{p_{i}}^{-} t^{i}} \prod_{j=0}^{i-1}\left(\Gamma_{p_{i}}-\Gamma_{p_{j}}\right)\left(\sum_{k=0}^{i} \frac{\left.c_{i}^{S^{1}}(M)\right|_{p_{k}}}{\prod_{j \in\{0, \ldots, \hat{k}, \ldots i\}}\left(\Gamma_{p_{k}}-\Gamma_{p_{j}}\right)}\right) \widetilde{\alpha}_{i} .
\end{gathered}
$$

(In particular, $\widetilde{\alpha}_{i} \in H^{2 i}(M ; \mathbb{Z})$ for all $i$.)

Proof. As an $H^{*}\left(\mathbb{C P}^{\infty} ; \mathbb{Z}\right)=\mathbb{Z}[t]$ module, $H_{S^{1}}^{*}(M ; \mathbb{Z})$ is freely generated by the classes $1, \alpha_{1}, \ldots, \alpha_{n}$, where

$$
\alpha_{i}=\Lambda_{p_{i}}^{-} \prod_{j=0}^{i-1} \frac{c_{1}^{S^{1}}(M)-\Gamma_{p_{j}} t}{\Gamma_{p_{i}}-\Gamma_{p_{j}}} \in H_{S^{1}}^{2 i}(M ; \mathbb{Z}) \quad \forall i .
$$


The image of $\alpha_{i}$ under the restriction map from equivariant cohomology to ordinary cohomology is

$$
\widetilde{\alpha}_{i}=\Lambda_{p_{i}}^{-} \frac{c_{1}(M)^{i}}{\prod_{j=0}^{i-1}\left(\Gamma_{p_{i}}-\Gamma_{p_{j}}\right)} \in H^{2 i}(M ; \mathbb{Z}) .
$$

By formality, $H^{*}(M ; \mathbb{Z})$ is freely generated (as a group) by $\widetilde{\alpha}_{0}, \ldots, \widetilde{\alpha}_{n}$.

Applying Proposition 3.9 to the reversed circle action with moment map $-\Phi$, we may define

$$
\beta_{n-i}=\Lambda_{p_{i}}^{+} \prod_{j=i+1}^{n} \frac{c_{1}^{S^{1}}(M)-\Gamma_{p_{j}} t}{\Gamma_{p_{i}}-\Gamma_{p_{j}}} \in H_{S^{1}}^{2 n-2 i}(M ; \mathbb{Z}) ;
$$

let $\widetilde{\beta}_{n-i} \in H^{2 n-2 i}(M ; \mathbb{Z})$ be the image of $\beta_{n-i}$ under the restriction map from equivariant cohomology to ordinary cohomology. Note that

$$
\left.\left(\alpha_{i} \beta_{n-i}\right)\right|_{p_{k}}= \begin{cases}\Lambda_{p_{i}} & k=i, \\ 0 & \text { otherwise. }\end{cases}
$$

Therefore, Theorem 2.4 implies that

$$
\int_{M} \alpha_{i} \beta_{n-i}=1
$$

and so

$$
\int_{M} \widetilde{\alpha}_{i} \widetilde{\beta}_{n-i}=1
$$

Since $c_{i}(M)$ is a multiple of $\widetilde{\alpha}_{i}$, this implies that

$$
c_{i}(M)=\left(\int_{M} c_{i}(M) \widetilde{\beta}_{n-i}\right) \widetilde{\alpha}_{i}=\left(\int_{M} c_{i}^{S^{1}}(M) \beta_{n-i}\right) \widetilde{\alpha}_{i} .
$$

Since

$$
\left.\beta_{n-i}\right|_{p_{k}}= \begin{cases}\Lambda_{p_{i}}^{+} t^{n-i} \prod_{j=i+1}^{n} \frac{\Gamma_{p_{k}}-\Gamma_{p_{j}}}{\Gamma_{p_{i}}-\Gamma_{p_{j}}} & \text { if } 0 \leq k \leq i, \\ 0 & \text { if } i<k \leq n,\end{cases}
$$

the first equality follows immediately from Theorem 2.4 .

Finally, since $\int_{M} \alpha_{k} \beta_{n-k}=1$ for all $k \in\{0, \ldots, n\}$ by (3.20),

$$
\frac{\prod_{j \neq k}\left(\Gamma_{p_{k}}-\Gamma_{p_{j}}\right)}{\Lambda_{p_{k}}}=\int_{M} \prod_{j \neq k}\left(c_{1}^{S^{1}}(M)-\Gamma_{p_{j}}\right)=\int_{M} c_{1}(M)^{n} .
$$

Hence,

$$
\frac{\prod_{j \neq k}\left(\Gamma_{p_{k}}-\Gamma_{p_{j}}\right)}{\Lambda_{p_{k}}}=\frac{\prod_{j \neq l}\left(\Gamma_{p_{l}}-\Gamma_{p_{j}}\right)}{\Lambda_{p_{l}}} \quad \forall k, l \in\{0, \ldots, n\} .
$$

The second equality follows immediately.

Note that the equations for $c_{i}(M)$ above simplify in some cases. Not only is $c_{0}(M)=1$ and $c_{n}(M)=(n+1) \widetilde{\alpha}_{n}$, but also

$$
\begin{gathered}
c_{1}(M)=\frac{\Gamma_{p_{1}}-\Gamma_{p_{0}}}{\Lambda_{p_{1}}^{-}} \widetilde{\alpha}_{1}, \quad \text { and } \\
c_{2}(M)=\frac{\left.c_{2}^{S^{1}}(M)\right|_{p_{0}}\left(\Gamma_{p_{2}}-\Gamma_{p_{1}}\right)-\left.c_{2}^{S^{1}}(M)\right|_{p_{1}}\left(\Gamma_{p_{2}}-\Gamma_{p_{0}}\right)+\left.c_{2}^{S^{1}}(M)\right|_{p_{2}}\left(\Gamma_{p_{1}}-\Gamma_{p_{0}}\right)}{\left(\Gamma_{p_{1}}-\Gamma_{p_{0}}\right) \Lambda_{p_{2}}^{-} t^{2}} \widetilde{\alpha}_{2} .
\end{gathered}
$$


Example 3.22. Let the circle act on a symplectic manifold $(M, \omega)$ with moment map $\Phi: M \longrightarrow \mathfrak{t}^{*}$. Assume that the fixed set consists of four points $p_{0}, p_{1}, p_{2}$ and $p_{3}$ with weights $\{1,2,3\},\{1,-1, l\},\{1,-1,-l\}$, and $\{-1,-2,-3\}$, respectively. Then $\Lambda_{p_{1}}^{-}=-1, \Lambda_{p_{2}}^{-}=l, \Gamma_{p_{0}}=6, \Gamma_{p_{1}}=-\Gamma_{p_{2}}=l,\left.c_{2}(M)\right|_{p_{0}}=11 t^{2}$, and $\left.c_{2}(M)\right|_{p_{1}}=\left.c_{2}(M)\right|_{p_{2}}=-t^{2}$. Therefore, Corollary 3.19 (and the equations above) immediately imply that

$$
\begin{aligned}
H^{*}(M ; \mathbb{Z}) & =\mathbb{Z}[x, y] /\left(x^{2}-\frac{2(l+6)}{(6-l)^{2}} y, y^{2}\right), \quad \text { and } \\
c(M) & =1+(6-l) x+\frac{24}{6-l} y+4 x y,
\end{aligned}
$$

where $x$ has degree 2 and $y$ has degree 4 .

The final lemma gives the relationship between the moment image and the sum of the weights at fixed components.

Lemma 3.23. Let the circle act on a compact symplectic manifold $(M, \omega)$ with moment map $\Phi: M \longrightarrow \mathbb{R}$. If $H^{2}(M ; \mathbb{R})=\mathbb{R}$, then for all fixed components $F$ and $F^{\prime}$

$$
\Gamma_{F}>\Gamma_{F^{\prime}} \quad \text { exactly if } \Phi(F)<\Phi\left(F^{\prime}\right) .
$$

Proof. By Lemma 2.7, there exists an equivariant extension $w \in H_{S^{1}}^{2}(M ; \mathbb{R})$ of $[\omega]$ so that $\left.w\right|_{p}=-\Phi(p) t$ for all fixed points $p$. Since $H^{2}(M ; \mathbb{R})=\mathbb{R}$ and $[\omega] \neq 0$, $c_{1}^{S^{1}}(M)=a w+b t$ for some real numbers $a$ and $b$. Therefore, given any fixed component $F, \Gamma_{F} t=\left.c_{1}^{S^{1}}(M)\right|_{p}=\left.(a w+b t)\right|_{p}=(-a \Phi(F)+b) t$ for all $p \in F$. On the other hand, if $F$ is the minimal fixed component and $F^{\prime}$ is the maximal fixed component, then $\Phi(F)<\Phi\left(F^{\prime}\right)$ and $\Gamma_{F}>0>\Gamma_{F^{\prime}}$. Therefore, $a>0$.

\section{The Case that the FIXed SET IS NOt Discrete}

We now return to the 6 -dimensional case. In this section, we prove Theorem 2 in the case that the fixed set is not discrete. In fact, in this case only the first two possibilities can arise.

Proposition 4.1. Let the circle act faithfully on a 6-dimensional compact symplectic manifold $(M, \omega)$ with moment map $\Phi: M \longrightarrow \mathbb{R}$. Assume that $H^{2}(M, \mathbb{R})=\mathbb{R}$ and the fixed set is not discrete. Then one of the following two statements is true:

(A) There is a subgroup $S^{1} \subset \mathrm{SU}(4)$ and an orientation-preserving diffeomorphism $f: M^{S^{1}} \longrightarrow\left(\mathbb{C P}^{3}\right)^{S^{1}}$ so that $\left.T(M)\right|_{M^{S^{1}}} \cong f^{*}\left(\left.T\left(\mathbb{C P}^{3}\right)\right|_{\left(\mathbb{C P}^{3}\right)^{S^{1}}}\right)$.

(B) There is a subgroup $S^{1} \subset \mathrm{SO}(5)$ and an orientation-preserving diffeomorphism $f: M^{S^{1}} \longrightarrow \widetilde{G}_{2}\left(\mathbb{R}^{5}\right)^{S^{1}}$ so that $\left.T(M)\right|_{M^{S^{1}}} \cong f^{*}\left(\left.T\left(\widetilde{G}_{2}\left(\mathbb{R}^{5}\right)\right)\right|_{\widetilde{G}_{2}\left(\mathbb{R}^{5}\right)^{S^{1}}}\right)$.

Remark 4.2. The manifolds described above do not contain an isolated fixed point with three distinct weights. A fortiori, they do not contain a pair of isotropy spheres which intersect in two points.

Remark 4.3. In fact, after possibly multiplying the standard symplectic form $\widehat{\omega}$ on $\mathbb{C P}^{3}$ (or $\widetilde{G}_{2}\left(\mathbb{R}^{5}\right)$ ) by a constant, we may assume that $f$ is a symplectomorphism on each fixed component. To see this, first note that the argument in Remark $3.17 \mathrm{im}-$ plies that $f^{*}\left(\left.[\widehat{\omega}]\right|_{\widehat{M}^{S^{1}}}\right)=\left.[\omega]\right|_{M^{S^{1}}}$. If each fixed component is at most 2-dimensional, this immediately implies that there exists a symplectomorphism $f^{\prime}: M^{S^{1}} \longrightarrow \widehat{M}^{S^{1}}$ 
which is homotopic to $f$. Otherwise, the claim follows from [Del]; see Case IV below.

We will need the following lemma to analyze the isotropy submanifolds which might arise.

Lemma 4.4. Let the circle act on a 4-dimensional compact symplectic manifold $(Z, \sigma)$ with moment map $\Psi: Z \longrightarrow \mathbb{R}$.

(1) If $Z^{S^{1}}$ consists of a minimal surface $\Sigma$ and one point, then $e\left(N_{\Sigma}\right)$, the Euler class of the normal bundle to $\Sigma$, is the positive generator of $H^{2}(\Sigma ; \mathbb{Z})$.

(2) If $Z^{S^{1}}$ consists of a minimal surface $\Sigma$ and two points, then $e\left(N_{\Sigma}\right)=0$.

(3) If $Z^{S^{1}}$ consists of a minimal surfaces $\Sigma$ and a maximal surface $\Sigma^{\prime}$, then there exists $a \in \mathbb{Z}$ so that $e\left(N_{\Sigma}\right)=$ au and $e\left(N_{\Sigma^{\prime}}\right)=-a u^{\prime}$. Here, $u$ and $u^{\prime}$ are the positive generators of $H^{2}(\Sigma ; \mathbb{Z})$ and $H^{2}\left(\Sigma^{\prime} ; \mathbb{Z}\right)$, respectively.

Proof. By dividing out by a finite subgroup, we may assume that the action is faithful. Let $e_{S^{1}}\left(N_{\Sigma}\right)=t+e\left(N_{\Sigma}\right)=t+a u$, where $H^{*}\left(\mathbb{C P}^{\infty} ; \mathbb{Z}\right)=\mathbb{Z}[t]$. Recall that every isotropy sphere contains exactly two isolated fixed points.

In case (1), since $\Psi$ is a Morse-Bott function the fixed point must have index 4 . Since there is only one isolated fixed point, there are no isotropy spheres. Hence, both weights at the isolated fixed point are -1 . Since the degree of 1 is $0<4$, $\int_{Z} 1=0$. Hence Theorem 2.4 and Remark 2.5 imply that $-a+1=0$, that is, $a=1$.

In case (2), since $\Psi$ is a Morse-Bott function, one fixed point must have index 2 and one must have index 4 . There are no isotropy spheres except possibly one joining these two points. Therefore, the weights at these points are $\{-1, l\}$ and $\{-1,-l\}$ for some natural number $l$. Since $\int_{Z} 1=0$, Theorem 2.4 implies that $-a-\frac{1}{l}+\frac{1}{l}=0$, that is, $a=0$.

In case (3), let $e_{S^{1}}\left(N_{\Sigma^{\prime}}\right)=-t+e\left(N_{\Sigma^{\prime}}\right)=-t+b u^{\prime}$. Since $\int_{Z} 1=0$, Theorem 2.4 implies that $-a-b=0$.

We will spend the remainder of this section proving Proposition 4.1. Let the circle act on a symplectic manifold $(M, \omega)$ with moment map $\Phi: M \longrightarrow \mathbb{R}$; assume that the conditions of the proposition are satisfied. By Remark $1.1, H^{2 i}(M ; \mathbb{R})=\mathbb{R}$ for all $i$ such that $0 \leq 2 i \leq 6$.

Let $N \subset M^{\mathbb{Z}_{k}}$ be any 4-dimensional isotropy submanifold. Since $\left.\Phi\right|_{N}$ is a MorseBott function and the critical sets of $\left.\Phi\right|_{N}$ are the fixed sets, $N^{S^{1}}$ must contain at least two fixed components, and at least one component must have index 0 or 2 .

Finally, notice that the action obtained by reversing the circle action and replacing $\Phi$ by $-\Phi$ also satisfies the assumptions of Proposition 4.1. Moreover, if this new action satisfies the conclusions of the proposition, then the original action does as well. Therefore, we can replace $\Phi$ by $-\Phi$ at any time. Given this symmetry, we only need to consider four cases.

Case I. The fixed set is discrete except for one minimal fixed surface.

Since $\Phi$ is a perfect Morse-Bott function, Poincaré duality implies that the fixed set consists of a minimal fixed sphere $\Sigma_{0}$ and two fixed points $p_{2}$ and $p_{3}$ of index 4 and 6 , respectively. Since every vector bundle over a surface splits, the normal bundle to $\Sigma_{0}$ is the direct sum of two line bundles with equivariant Euler classes 
$m t+a u$ and $n t+b u$, where $m$ and $n$ are natural numbers, $a$ and $b$ are integers, and $u$ is the positive generator of $H^{2}\left(\Sigma_{0} ; \mathbb{Z}\right)$.

Assume first that there is a 4-dimensional isotropy submanifold $N \subset M^{\mathbb{Z}_{m}}$ which contains both $p_{2}$ and $p_{3}$. Since the intersection of any two distinct 4-dimensional isotropy submanifolds is 2-dimensional, each component of the intersection must be either a fixed surface or an isotropy sphere. Hence, every 4-dimensional isotropy submanifold must contain both fixed points. So there exists a natural number $l$ which is a multiple of both $m$ and $n$ so that the weights at $p_{2}$ and $p_{3}$ are $\{-m,-n, l\}$ and $\{-m,-n,-l\}$, respectively. Applying Lemma 4.4 to the isotropy submanifold $N \subset M^{\mathbb{Z}_{m}}$, we see that $a=0$. Moreover, Lemma 2.6 implies that $2 n=0 \bmod m$. Since $m \neq 1$ and $n$ and $m$ are relatively prime, this implies that $m=2$ and $n=1$. Since the degree of 1 is $0<6$ and the degree of $c_{1}^{S^{1}}(M)$ is $2<6, \int_{M} 1=0$ and $\int_{M} c_{1}^{S^{1}}(M)=0$. Therefore, Theorem 2.4 and Remark 2.5 imply that

$$
-\frac{b}{2}+\frac{1}{2 l}-\frac{1}{2 l}=0 \quad \text { and } \quad(1-b)+\frac{l-3}{2 l}+\frac{l+3}{2 l}=0 .
$$

These equations simplify to $b=0$ and $b=2$, which is impossible.

Therefore, there is no 4-dimensional isotropy submanifold which contains both $p_{2}$ and $p_{3}$. If $m \neq 1$, then this implies that $M^{\mathbb{Z}_{m}}$ contains a 4-dimensional isotropy submanifold $N$ which contains $\Sigma_{0}$ and exactly one fixed point. By Lemma 4.4, this implies that $a=1$. A nearly identical argument implies that if $n \neq 1$, then $b=1$. Moreover, if there exist any isotropy spheres, then each one must contain $p_{2}$ and $p_{3}$. Hence, there exists a natural number $l$ so that the weights at $p_{2}$ and $p_{3}$ are $\{-m,-m, l\}$ and $\{-n,-n,-l\}$, respectively. Since $\int_{M} 1=0$ and $\int_{M} c_{1}^{S^{1}}(M)=0$, Theorem 2.4 and Remark 2.5 imply that

$$
\begin{gathered}
-\left(\frac{a}{m^{2} n}+\frac{b}{m n^{2}}\right)+\frac{1}{l m^{2}}-\frac{1}{l n^{2}}=0, \text { and } \\
\left(\frac{2}{m n}-\frac{a}{m^{2}}-\frac{b}{n^{2}}\right)+\left(\frac{1}{m^{2}}-\frac{2}{l m}\right)+\left(\frac{1}{n^{2}}+\frac{2}{l n}\right)=0 .
\end{gathered}
$$

Assume first that $l \geq m$ and $l \geq n$. If $l=1$, then $m=n=1$ as well. Otherwise, applying Lemma 2.6 to the isotropy sphere $N \subset M^{\mathbb{Z}_{l}}$ implies that $m=n \bmod l$, and hence again $m=n$. Equations (4.5) and (4.6) now simplify to $a+b=0$ and $a+b=4$, respectively. This is impossible.

Assume next that $m>l$ and $m \geq n$. As we saw in the third paragraph of this proof, the fact that $m \neq 1$ implies that $a=1$. Moreover, applying Lemma 2.6] to the isotropy submanifold $N \subset M^{\mathbb{Z}_{m}}$ implies that $n=l \bmod m$, and hence $n=l$. Equations (4.5) and (4.6) now simplify to $b n+m=0$ and $b=3$, respectively. Since $n$ and $m$ are both positive, this is impossible.

Finally, assume that $n>m$ and $n>l$. As we saw in the third paragraph of this proof, the fact that $n \neq 1$ implies that $b=1$. Moreover, applying Lemma 2.6 to the isotropy submanifold $N \subset M^{\mathbb{Z}_{n}}$ implies that $m+l=0 \bmod n$, and hence $n=m+l$. Equation (4.5) now simplifies to $a=1$. In sum, $a=b=1$ and $n=m+l$. Comparing with Example 2.8, statement (A) is true for the action

$$
\lambda \cdot\left[x_{0}, x_{1}, x_{2}, x_{3}\right]=\left[x_{0}, x_{1}, \lambda^{m} x_{2}, \lambda^{n} x_{3}\right] .
$$

Case J. The fixed set is discrete except for one non-extremal fixed surface.

Since $\Phi$ is a perfect Morse-Bott function, the fixed set consists of a point $p_{0}$ of index 0 , a surface $\Sigma_{1}$ of index 2 , and a point $p_{3}$ of index 6 . Since $\Sigma_{1}$ has index 
2 , the normal bundle to $\Sigma_{1}$ is the direct sum of two line bundles with equivariant Euler classes $m t+a u$ and $-n t+b u$, where $m$ and $n$ are natural numbers, $a$ and $b$ are integers, and $u$ is the positive generator of $H^{2}\left(\Sigma_{1} ; \mathbb{Z}\right)$. After possibly replacing $\Phi$ by $-\Phi$, we may assume that $m \geq n$.

Assume first that $m=n=1$. Let $\left\{l_{1}, l_{2}, l_{3}\right\}$ be the weights at $p_{0}$, where $l_{1} \geq l_{2} \geq l_{3}$. Since $m=n=1$, if there exist any isotropy submanifolds, then each one has minimum $p_{0}$ and maximum $p_{3}$. Hence, the weights at $p_{3}$ are $\left\{-l_{1},-l_{2},-l_{3}\right\}$.

Let $\alpha=c_{1}^{S^{1}}(M)-\Gamma_{p_{0}} t \in H_{S^{1}}^{2}(M ; \mathbb{Z})$. Since $\left.\alpha\right|_{p_{0}}=0$, Proposition 2.2 implies that $\left.\alpha\right|_{\Sigma_{1}}$ is a multiple of $-t+b u$, which is the equivariant Euler class of the negative normal bundle at $\Sigma_{1}$. On the other hand,

$$
\begin{gathered}
\left.c_{1}^{S^{1}}(M)\right|_{\Sigma_{1}}=c_{1}\left(\Sigma_{1}\right)+(t+a u)+(-t+b u)=c_{1}\left(\Sigma_{1}\right)+(a+b) u, \quad \text { and } \\
\left.\alpha\right|_{\Sigma_{1}}=c_{1}\left(\Sigma_{1}\right)+(a+b) u-\Gamma_{p_{0}} t=c_{1}\left(\Sigma_{1}\right)+\left(a+b-\Gamma_{p_{0}} b\right) u+\Gamma_{p_{0}}(-t+b u) .
\end{gathered}
$$

Therefore, $c_{1}\left(\Sigma_{1}\right)=-\left(a+b-\Gamma_{p_{0}} b\right) u$. Applying the same argument to the moment map $-\Phi$ we see that $c_{1}\left(\Sigma_{1}\right)=-\left(a+b+\Gamma_{p_{3}} a\right) u$. Since $\Gamma_{p_{3}}=-\Gamma_{p_{0}} \neq 0$, this implies that $a=b$.

Since $\int_{M} c_{1}^{S^{1}}(M)=0$, Theorem 2.4 and Remark 2.5 imply that

$$
\frac{\Gamma_{p_{0}}}{\Lambda_{p_{0}}}-\Gamma_{p_{0}} a+\frac{\Gamma_{p_{3}}}{\Lambda_{p_{3}}}=0 .
$$

Since $\Lambda_{p_{0}}=-\Lambda_{p_{3}}$ and $\Gamma_{p_{0}}=-\Gamma_{p_{3}} \neq 0$, this simplifies to $a=\frac{2}{\Lambda_{p_{0}}}$. Hence, either $a=1, l_{1}=2$, and $l_{2}=l_{3}=1$; or $a=2$ and $l_{1}=l_{2}=l_{3}=1$. In either case, $c_{1}\left(\Sigma_{1}\right)=2$ and so $\Sigma_{1}$ is a sphere.

Suppose first that $a=b=1$, and $l_{1}=2$, and $l_{2}=l_{3}=n=m=1$. Comparing with Example 2.8, statement (A) is true for the circle action

$$
\lambda \cdot\left[x_{0}, x_{1}, x_{2}, x_{3}\right]=\left[x_{0}, \lambda x_{1}, \lambda x_{2}, \lambda^{2} x_{3}\right] .
$$

Suppose instead that $a=b=2$ and $l_{1}=l_{2}=l_{3}=n=m=1$. Comparing with Example 2.9, statement (B) is true for the circle action on $\widetilde{G}_{2}\left(\mathbb{R}^{5}\right)$ induced by the action on $\mathbb{R}^{5}=\mathbb{R} \times \mathbb{C}^{2}$ given by

$$
\lambda \cdot\left(t, x_{1}, x_{2}\right)=\left(t, x_{1}, \lambda x_{2}\right) .
$$

So instead, assume that $m \neq 1$. Then there is a 4-dimensional isotropy submanifold $N \subset M^{\mathbb{Z}_{m}}$ with minimum $\Sigma_{1}$ and maximum $p_{3}$. Since $\left.\Phi\right|_{N}$ is a perfect MorseBott function, Poincaré duality implies that $\Sigma_{1}$ is sphere. Moreover, Lemma 4.4 implies that $a=1$. Similarly, if $n \neq 1$, then there is a 4-dimensional isotropy submanifold $N \subset M^{\mathbb{Z}_{n}}$ with maximum $\Sigma_{1}$ and minimum $p_{0}$. If there exist other isotropy submanifolds, then each one has minimum $p_{0}$ and maximum $p_{3}$. Hence, there exists a natural number $l$ so that the weights at $p_{0}$ and $p_{3}$ are $\{n, n, l\}$ and $\{-m,-m,-l\}$, respectively.

Since $\int_{M} 1=0$ and $\int_{M} c_{1}^{S^{1}}(M)=0$, Theorem 2.4 and Remark 2.5 imply that

$$
\begin{aligned}
& \frac{1}{n^{2} l}+\left(\frac{1}{m^{2} n}-\frac{b}{m n^{2}}\right)-\frac{1}{m^{2} l}=0, \quad \text { and } \\
& \frac{2 n+l}{n^{2} l}-\left(\frac{1}{m^{2}}+\frac{b}{n^{2}}+\frac{2}{m n}\right)+\frac{2 m+l}{m^{2} l}=0 .
\end{aligned}
$$

Suppose first that $m \geq l$. Then applying Lemma 2.6 to the 4-dimensional isotropy submanifold $N \subset M^{\mathbb{Z}_{m}}$ implies that $n=l \bmod m$, and so $n=l$. Then 
equations (4.7) and (4.8) simplify to $n b=m$ and $3 n=b$. Hence, $3 n^{2}=m$; but this contradicts $n \geq m$.

So assume instead that $l>m$. Then applying Lemma 2.6 to the isotropy sphere $Z \subset M^{\mathbb{Z}_{l}}$ implies that $m+n=0 \bmod l$, and so $l=m+n$. Then equation (4.7) simplifies to $b=1$. In sum, $a=b=1$ and the weights at $p_{0}$ and $p_{3}$ are $\{n, n, m+n\}$ and $\{-m,-m,-(m+n)\}$. Comparing with Example 2.8, statement (A) is true for the circle action on $\mathbb{C P}^{3}$ given by

$$
\lambda \cdot\left[x_{0}, x_{1}, x_{2}, x_{3}\right]=\left[x_{0}, \lambda^{n} x_{1}, \lambda^{n} x_{2}, \lambda^{m+n} x_{3}\right] .
$$

Case K. The fixed set contains more than one fixed surface.

Since $\Phi$ is a perfect Morse-Bott function, the fixed set consists of two surfaces $\Sigma_{0}$ and $\Sigma_{2}$ of the same genus with indices 0 and 4 , respectively. Let $u$ and $u^{\prime}$ be the positive generators of $H^{2}\left(\Sigma_{0} ; \mathbb{Z}\right)$ and $H^{2}\left(\Sigma_{2} ; \mathbb{Z}\right)$, respectively. Our first claim is that $\Sigma_{0}$ and $\Sigma_{2}$ are spheres. Suppose on the contrary that $\Sigma_{0}$ and $\Sigma_{2}$ have positive genus. Then there exist classes $v_{1}$ and $v_{2} \in H^{1}\left(\Sigma_{0} ; \mathbb{Z}\right)$ so that $v_{1} v_{2}=u$. By Proposition 2.2, there exist classes $\alpha_{1}$ and $\alpha_{2} \in H_{S^{1}}^{1}(M ; \mathbb{Z})$ so that $\left.\alpha_{i}\right|_{\Sigma_{0}}=v_{i}$; let $\beta=\alpha_{1} \alpha_{2}$. On the one hand, $\left.\beta\right|_{\Sigma_{0}}=u$, and so Proposition 2.2 implies that $c_{1}^{S^{1}}(M) \in H_{S^{1}}^{2}(M ; \mathbb{Z})$ is a linear combination of $t$ and $\beta$. On the other hand, because $\left.\alpha_{i}\right|_{p}=0$ for dimensional reasons, $\left.\beta\right|_{p}=0$ for all fixed points $p$. Since $\Gamma_{\Sigma_{0}}>0>\Gamma_{\Sigma_{2}}$, this contradicts the facts that $\left.c_{1}^{S^{1}}(M)\right|_{p}=\Gamma_{F} t$ for all $p$ in a fixed component $F$. Hence, $\Sigma_{0}$ and $\Sigma_{2}$ are spheres.

The normal bundle over $\Sigma_{0}$ splits as the sum of two line bundles with equivariant Euler classes $m t+a u$ and $n t+b u$, where $m$ and $n$ are natural numbers, and $a$ and $b$ are integers. Since every isotropy submanifold must have minimum $\Sigma_{0}$ and maximum $\Sigma_{2}$, the negative weights at $\Sigma_{2}$ are $-m$ and $-n$. Hence, the normal bundle over $\Sigma_{2}$ splits as the sum of two line bundles with equivariant Euler classes $-m t+c u^{\prime}$ and $-n t+d u^{\prime}$, where $c$ and $d$ are integers.

Since the degree of $\left(c_{1}^{S^{1}}\right)^{2}-2 c_{2}^{S^{1}}$ is $4<6, \int_{M} 1=0, \int_{M} c_{1}^{S^{1}}=0$, and $\int_{M}\left(\left(c_{1}^{S^{1}}\right)^{2}-2 c_{2}^{S^{1}}\right)=0$. Therefore, Theorem 2.4 and Remark 2.5 imply that

$$
\begin{gathered}
-\left(\frac{a}{m^{2} n}+\frac{b}{m n^{2}}\right)+\left(\frac{c}{m^{2} n}+\frac{d}{m n^{2}}\right)=0, \\
\left(\frac{2}{m n}-\frac{a}{m^{2}}-\frac{b}{n^{2}}\right)+\left(\frac{2}{m n}-\frac{c}{m^{2}}-\frac{d}{n^{2}}\right)=0, \quad \text { and } \\
\left(\frac{a}{n}+\frac{b}{m}-\frac{n a}{m^{2}}-\frac{m b}{n^{2}}\right)-\left(\frac{c}{n}+\frac{d}{m}-\frac{n c}{m^{2}}-\frac{m d}{n^{2}}\right)=0 .
\end{gathered}
$$

We may assume that $m \geq n$. If $m \neq 1$, then applying Lemma 2.6 to the isotropy submanifold $N \subset M^{\mathbb{Z}_{m}}$ implies that $2 n=0 \bmod m$. Since $m$ and $n$ are relatively prime, this implies that $m=2$ and $n=1$. Hence $m \leq 2$ and $n=1$.

Assume first that $m=n=1$. Then equations (4.9) and (4.10) simplify to $a+b=c+d$ and $a+b+c+d=4$. In sum, $m=n=1$ and $a+b=c+d=2$. Comparing with Example 2.8, statement (A) is true for the circle action on $\mathbb{C P}^{3}$ given by

$$
\lambda \cdot\left[x_{0}, x_{1}, x_{2}, x_{3}\right]=\left[x_{0}, x_{1}, \lambda x_{2}, \lambda x_{3}\right] .
$$

So assume instead that $m=2$ and $n=1$. Applying Lemma 4.4 to the isotropy submanifold $N \subset M^{\mathbb{Z}_{2}}$, we see that $a=-c$. Therefore, equations (4.9), (4.10), and (4.11) simplify to $a+b=d, b+d=2$, and $a+d=b$, respectively. In sum, $m=2$, 
$n=1, a=c=0$ and $b=d=1$. Comparing with Example 2.9, statement (B) is true for the circle action on $\widetilde{G}_{2}\left(\mathbb{R}^{5}\right)$ induced by the action on $\mathbb{R}^{5}=\mathbb{R} \times \mathbb{C}^{2}$ given by

$$
\lambda \cdot\left(t, x_{1}, x_{2}\right)=\left(t, \lambda x_{1}, \lambda x_{2}\right) .
$$

Case L. The fixed set contains a four-dimensional component.

Let $F$ be a four-dimensional component of the fixed set. Since $F$ is symplectic, $H^{2}(F ; \mathbb{R}) \neq 0$. Since $\Phi$ is a perfect Morse-Bott function, this implies that $M^{S^{1}}$ consists of $F$ and one isolated fixed point. By Delzant [Del] - after possibly rescaling $\omega$ and reversing the circle action $-(M, \omega)$ is equivariantly symplectomorphic to $\mathbb{C P}^{3}$ with its standard symplectic form and the circle action given by

$$
\lambda \cdot\left[x_{0}, x_{1}, x_{2}, x_{3}\right]=\left[x_{0}, x_{1}, x_{2}, \lambda x_{3}\right] .
$$

A fortiori, statement (A) is true.

This completes the proof of Proposition 4.1

\section{The Case that the FiXed Set is Discrete: Defining the Multigraph}

In the remainder of the paper, we prove Theorem 2 in the case that the fixed set is discrete. In particular, in this section, we assume that the fixed set is discrete and define an associated multigraph which is labeled: a real number and an even integer are associated to each vertex, and a natural number $l_{e}$ (the length of $e$ ) is associated to each edge $e$.

Let the circle act faithfully on a 6-dimensional compact symplectic manifold $(M, \omega)$ with moment map $\Phi: M \longrightarrow \mathbb{R}$; assume that the fixed set $M^{S^{1}}$ is discrete. We define an associated labeled multigraph as follows: The vertex set is the fixed set $M^{S^{1}}$; each fixed point $p$ is labeled by its moment image $\Phi(p)$ and its index $2 \lambda_{p}$. Given distinct $p$ and $q \in M^{S^{1}}$, let $E_{p q}$ denote the set of edges joining $p$ and $q$. If $\Phi(p) \leq \Phi(q)$, then there is a (unique) edge $e \in E_{p q}$ of length $k \neq 1$ exactly if the following are all true:

(1) $p$ and $q$ lie in the same component $N \subset M^{\mathbb{Z}_{k}}$.

(2) $k$ is one of the weights at $p ;-k$ is one of the weights at $q$.

(3) The index of $\left.\Phi\right|_{N}$ at $q$ is equal to 2 plus the index of $\left.\Phi\right|_{N}$ at $p$.

(In particular, $\Phi(p)<\Phi(q)$.) We say the edge $e \in E_{p q}$ has minimum $p$ and maximum $q$.

A multigraph contains multiple edges if there are several edges with the same minimum and maximum. The multigraph is simple if there are no multiple edges.

Example 5.1. Fix natural numbers $m, n$, and $k$, and consider the circle action on $\mathbb{C P}^{3}$ given by

$$
\lambda \cdot\left[x_{0}, x_{1}, x_{2}, x_{3}\right]=\left[x_{0}, \lambda^{m} x_{1}, \lambda^{m+n} x_{2}, \lambda^{m+n+k} x_{3}\right] .
$$

There is a unique fixed point $p_{i}$ of index $2 i$ for all $i$ such that $0 \leq 2 i \leq 6$. If $1 \notin\{m, n, k\}$, the associated multigraph is the complete graph on $\left\{p_{0}, p_{1}, p_{2}, p_{3}\right\}$; moreover, $l_{01}=m, l_{12}=n, l_{23}=k, l_{02}=m+n, l_{13}=n+k$, and $l_{03}=m+n+k$, where $l_{i j}$ is the length of the edge from $p_{i}$ to $p_{j}$. (In contrast if, for example, $m=1$, then there is no edge joining $p_{0}$ to $p_{1}$.) 
This multigraph has a number of nice properties. First, the labeled multigraph determines the weights at every fixed point. Second, the weights at the minimum and maximum of any edge are equal modulo its length. Third, if two edges have the same minimum and maximum, then their lengths are relatively prime. To prove the first property, we need the following proposition to analyze the isotropy submanifolds which might arise; see [Kar].

Proposition 5.2 (Karshon). Let the circle act faithfully on a 4-dimensional compact connected symplectic manifold $(N, \sigma)$ with moment map $\Psi: N \longrightarrow \mathbb{R}$; assume that the fixed set $N^{S^{1}}$ is discrete. The multiplicity of the weight +1 at the minimum is the number of fixed points of index 2 with negative weight-1. Similarly, the multiplicity of the weight -1 at the maximum is the number of fixed points of index 2 with positive weight +1 .

We are now ready to prove the properties described above.

Lemma 5.3. Let the circle act faithfully on a 6-dimensional compact symplectic manifold $(M, \omega)$ with moment map $\Phi: M \longrightarrow \mathbb{R}$. Assume that the fixed set $M^{S^{1}}$ is discrete and consider the associated labeled multigraph. Given any fixed point $p$, there are at most $\lambda_{p}$ edges with maximum $p$ and at most $3-\lambda_{p}$ edges with minimum $p$. Moreover, the multiset of weights at $p$ is the multiset of $\lambda_{p}$ negative integers and $3-\lambda_{p}$ positive integers obtained by adding 1 and -1 with appropriate multiplicity to

$$
\left\{\operatorname{sign}(\Phi(q)-\Phi(p)) l_{e} \mid q \in M^{S^{1}} \text { and } e \in E_{p q}\right\} .
$$

Proof. It is enough to show that for all $k>1$, the multiplicity of the weight $k$ at $p$ is the number of edges $e \in E$ with minimum $p$ and length $k$ (the analogous claim then holds for all $k<-1$ ).

Let $N$ be the component of $M^{\mathbb{Z}_{k}}$ which contains $p$. If $\operatorname{dim}(N)=0$ or $\operatorname{dim}(N)=2$, then the claim above is obvious. On the other hand, if $\operatorname{dim}(N)=4$, then the claim follows immediately from Proposition 5.2 where we consider the faithful $S^{1} / \mathbb{Z}_{k^{-}}$ action on $N$.

Lemma 5.4. Let the circle act faithfully on a 6-dimensional compact symplectic manifold $(M, \omega)$ with moment map $\Phi: M \longrightarrow \mathbb{R}$. Assume that the fixed set $M^{S^{1}}$ is discrete and consider the associated labeled multigraph. Given any edge $e \in E_{p q}$, the weights at $p$ and $q$ are equal modulo $l_{e}$.

Proof. Since $p$ and $q$ are contained in the same component of $M^{\mathbb{Z}_{l_{e}}}$, the claim follows immediately from Lemma 2.6 .

Lemma 5.5. Let the circle act faithfully on a 6-dimensional compact symplectic manifold $(M, \omega)$ with moment map $\Phi: M \longrightarrow \mathbb{R}$. Assume that the fixed set $M^{S^{1}}$ is discrete and consider the associated labeled multigraph. Given any distinct edges $e$ and $e^{\prime}$ in $E_{p q}$, the lengths $l_{e}$ and $l_{e^{\prime}}$ are relatively prime.

Proof. Assume not; let $k \neq 1$ be the greatest common divisor of $l_{e}$ and $l_{e^{\prime}}$. We may also assume that $\Phi(p) \leq \Phi(q)$ and that $l_{e} \geq l_{e^{\prime}}$. Let $N \subset M^{\mathbb{Z}_{l_{e}}}$ and $N^{\prime} \subset M^{\mathbb{Z}_{l^{\prime}}}$ be the components which contain $p$ and $q$. The component $Z \subset M^{\mathbb{Z}_{k}}$ which contains $p$ also contains $N$ and $N^{\prime}$, and hence $q$. By construction, the weights at $p$ and $q$ are $\left\{l_{e}, l_{e^{\prime}}, a\right\}$ and $\left\{-l_{e},-l_{e^{\prime}}, b\right\}$, respectively, for some integers $a$ and $b$. Since the action is faithful, $a$ is not a multiple of $k$; therefore, $l_{e^{\prime}} \neq a \bmod l_{e}$. On the other 
hand, by Lemma 2.6 the weights at $p$ are equal to the weights at $q$ modulo $l_{e}$. Since $l_{e}=-l_{e} \bmod l_{e}$, this implies that $2 l_{e^{\prime}}=0 \bmod l_{e}$. Since $l_{e} \geq l_{e^{\prime}}$, this implies that $l_{e}$ is a multiple of $l_{e^{\prime}}$, that is, $l_{e^{\prime}}=k$. But then the index of $\left.\Phi\right|_{N^{\prime}}$ at $p$ is 0 , while the index of $\left.\Phi\right|_{N^{\prime}}$ at $q$ is 4 . This is impossible by assumption (3) in the definition of the multigraph.

Remark 5.6. We will sometimes add edges of length 1 to $G$ in order to reduce the number of cases that we need to consider. (For example, this allows us to drop the condition $1 \notin\{m, n, k\}$ in Example 5.1.) Lemmas 5.4 and 5.5 clearly still hold for this "extended" multigraph. Moreover, as long as we add these edges so that there are still at most $\lambda_{p}$ edges with maximum $p$ and $3-\lambda_{p}$ edges with minimum $p$, Lemma 5.3 still holds.

\section{The CASE that THE FiXed SET IS DiscRete AND THE ASSOciated MULTIGRAPH IS SIMPLE}

In this section, we prove Theorem 2 in the case that the fixed set is discrete and the associated multigraph is simple, that is, contains no multiple edges. In fact, in this case only the first two possibilities can arise.

Proposition 6.1. Let the circle act faithfully on a 6-dimensional compact symplectic manifold $(M, \omega)$ with moment map $\Phi: M \longrightarrow \mathbb{R}$. Assume that $H^{2}(M ; \mathbb{R})=\mathbb{R}$, the fixed set is discrete, and the associated multigraph is simple. Then one of the following two statements is true:

(A) There is a subgroup $S^{1} \subset \mathrm{SU}(4)$ and bijection $f: M^{S^{1}} \longrightarrow\left(\mathbb{C P}^{3}\right)^{S^{1}}$ so that $\left.T(M)\right|_{M^{S^{1}}} \cong f^{*}\left(\left.T\left(\mathbb{C P}^{3}\right)\right|_{\left(\mathbb{C P}^{3}\right)^{S^{1}}}\right)$.

(B) There is a subgroup $S^{1} \subset \mathrm{SO}(5)$ and bijection $f: M^{S^{1}} \longrightarrow \widetilde{G}_{2}\left(\mathbb{R}^{5}\right)^{S^{1}}$ so that $\left.T(M)\right|_{M^{S^{1}}} \cong f^{*}\left(\left.T\left(\widetilde{G}_{2}\left(\mathbb{R}^{5}\right)\right)\right|_{\widetilde{G}_{2}\left(\mathbb{R}^{5}\right)^{S^{1}}}\right)$.

Remark 6.2. Since the associated multigraph is simple, the manifolds described above do not contain a pair of isotropy spheres which intersect in two points.

Our proof relies heavily on the following technical lemma.

Lemma 6.3. Fix a natural number $l_{i j} \geq 1$ for each pair $\{i, j\} \subset\{0,1,2,3\}$. Assume that the multisets $\left\{\operatorname{sign}(k-i) l_{i k} \mid k \neq i\right\}$ and $\left\{\operatorname{sign}(k-j) l_{j k} \mid k \neq j\right\}$ are equal modulo $l_{i j}$ for each such pair. Then one of the following three statements is true:

(a) $l_{02} \leq l_{01}+l_{12}, \quad l_{13} \leq l_{12}+l_{23}, \quad l_{03} \leq l_{01}+l_{13}$, and $l_{03} \leq l_{02}+l_{23}$.

(b) $l_{01}=l_{23}, \quad l_{02} \leq l_{12}+l_{03}, l_{13} \leq l_{12}+l_{03}$, and $l_{03} \leq l_{01}+l_{12}$.

(c) $l_{02}=l_{13}$ and $l_{03} \leq l_{12}$.

Proof of Proposition 6.1. By Remark 1.1, $H^{2 i}(M ; \mathbb{R})=\mathbb{R}$ for all $i$ such that $0 \leq$ $2 i \leq 6$. Hence, since $\Phi$ is a perfect Morse function, there is exactly one fixed point $p_{i}$ of index $2 i$ for all $i$ such that $0 \leq 2 i \leq 6$. By Proposition 3.4 . $\Phi\left(p_{i}\right)<\Phi\left(p_{j}\right)$ exactly if $i<j$.

By assumption, the associated labeled multigraph $G$ is simple. Add edges of length 1 so that $G$ is a complete graph. Then for each vertex $p_{i}$ there are exactly $i$ edges with maximum $p_{i}$ and $3-i$ edges with minimum $p_{i}$. (See Remark 5.6.) By Lemma 5.3 the multiset of weights at $p_{i}$ is $\left\{\operatorname{sign}(k-i) l_{i k} \mid k \neq i\right\}$, where $l_{i k}$ denotes the length of the edge $\{i, k\}$. By Lemma 5.4, the weights at $p_{i}$ and $p_{j}$ are 
equal modulo $l_{i j}$ for each pair $\{i, j\} \subset\{0,1,2,3\}$. Therefore, Lemma 6.3 implies that one of the three statements (a), (b), or (c) is true. Finally, since $c_{1}^{S^{1}}(M)$ has degree $2<6, \int_{M} c_{1}^{S^{1}}(M)=0$. Hence, Theorem 2.4 (together with Remark 2.5) implies that

$$
\frac{l_{01}+l_{02}+l_{03}}{l_{01} l_{02} l_{03}}+\frac{l_{01}-l_{12}-l_{13}}{l_{01} l_{12} l_{13}}+\frac{l_{23}-l_{02}-l_{12}}{l_{02} l_{12} l_{23}}+\frac{l_{03}+l_{13}+l_{23}}{l_{03} l_{13} l_{23}}=0 .
$$

First assume that statement (a) is true. Rewrite equation (6.4) as

$$
\frac{l_{01}+l_{12}-l_{02}}{l_{01} l_{12} l_{02}}+\frac{l_{12}+l_{23}-l_{13}}{l_{12} l_{23} l_{13}}+\frac{l_{01}+l_{13}-l_{03}}{l_{01} l_{13} l_{03}}+\frac{l_{02}+l_{23}-l_{03}}{l_{02} l_{23} l_{03}}=0 .
$$

Since all the lengths are positive, this implies that the inequalities in statement (a) are equalities, that is, $l_{02}=l_{01}+l_{12}, \quad l_{13}=l_{12}+l_{23}$, and $l_{03}=l_{01}+l_{12}+l_{23}$. Comparing with Example 2.8, the weights at the fixed points agree with those associated to the circle action on $\mathbb{C P}^{3}$ given by

$$
\lambda \cdot\left[x_{0}, x_{1}, x_{2}, x_{3}\right]=\left[x_{0}, \lambda^{l_{01}} x_{1}, \lambda^{l_{01}+l_{12}} x_{2}, \lambda^{l_{01}+l_{12}+l_{23}} x_{3}\right] .
$$

Hence statement (A) is true.

Now assume that statement (b) is true. Since $l_{01}=l_{23}$, we can rewrite equation (6.4) as

$$
\frac{l_{12}+l_{03}-l_{02}}{l_{12} l_{03} l_{02}}+\frac{l_{12}+l_{03}-l_{13}}{l_{12} l_{03} l_{13}}+2 \frac{l_{01}+l_{12}-l_{03}}{l_{01} l_{12} l_{03}}=0 .
$$

Together with the inequalities in statement (b), this implies that $l_{01}=l_{23}, \quad l_{03}=$ $l_{01}+l_{12}$, and $l_{02}=l_{13}=l_{01}+2 l_{12}$. Comparing with Example 2.9, the weights at the fixed points agree with those associated to the circle action on $\widetilde{G}_{2}\left(\mathbb{R}^{5}\right)$ induced by the action on $\mathbb{R}^{5}=\mathbb{R} \times \mathbb{C}^{2}$ given by

$$
\lambda \cdot\left(t, x_{1}, x_{2}\right)=\left(t, \lambda^{l_{12}} x_{1}, \lambda^{l_{01}+l_{12}} x_{2}\right) .
$$

Hence statement (B) is true.

Finally, assume that statement (c) is true. Since $l_{02}=l_{13}$, we can rewrite equation (6.4) as

$$
\frac{2}{l_{02} l_{03}}+\frac{2}{l_{02} l_{12}}+\frac{l_{12}-l_{03}}{l_{01} l_{12} l_{03}}+\frac{l_{12}-l_{03}}{l_{23} l_{12} l_{03}}=0 .
$$

This contradicts the fact that $l_{03} \leq l_{12}$.

We will spend the remainder of this section proving Lemma 6.3 Fix a natural number $l_{i j}$ for each pair $\{i, j\} \subset\{0,1,2,3\}$; assume that they satisfy the assumptions of the lemma. We will think of $l_{i j}$ as labeling the edge $e_{i j}$ on the complete graph $G$ on $\left\{p_{0}, p_{1}, p_{2}, p_{3}\right\}$. We say that $e_{i j}$ is longer than $e_{m n}$ if either $l_{i j}>l_{m n}$ or if $l_{i j}=l_{m n}$ and $e_{i j}$ appears before $e_{m n}$ on this list: $e_{01}, e_{23}, e_{12}, e_{02}, e_{13}, e_{03} 6$

Fix an edge $e_{i j}$, and let $\{m, n\}=\{1,2,3,4\} \backslash\{i, j\}$. We say that $e_{i j}$ is regular if $\operatorname{sign}(m-i) l_{i m}=\operatorname{sign}(m-j) l_{j m} \bmod l_{i j}$ and $\operatorname{sign}(n-i) l_{i n}=\operatorname{sign}(n-j) l_{j n} \bmod l_{i j}$. In contrast, we say that $e_{i j}$ is goofy 7 if $\operatorname{sign}(m-i) l_{i m}=\operatorname{sign}(n-j) l_{j n} \bmod l_{i j}$ and $\operatorname{sign}(n-i) l_{i n}=\operatorname{sign}(m-j) l_{j m} \bmod l_{i j}$. Since $l_{i j}=-l_{i j} \bmod l_{i j}$, every edge must be regular, goofy, or both.

\footnotetext{
${ }^{6}$ We break the ties in this way to simplify the argument. For example, the arguments are very similar in the case that $l_{01}=l_{12}$ and the case that $l_{01}>l_{02}$.

7 These terms are borrowed from surfing, where they describe which foot is in front. We chose them to emphasize that there are exactly two possibilities, that one of them is more common, and that both are equally valid.
} 
We will need the following fact.

Lemma 6.5. If $l_{03}=l_{13}$, then $l_{03} \leq l_{02}+l_{23}$. Similarly, if $l_{03}=l_{23}$, then $l_{03} \leq l_{01}+l_{13}$.

Proof. Assume that $l_{03}=l_{13}$. If $e_{03}$ is regular, then $l_{02}+l_{23}=0 \bmod l_{03}$, whereas if $e_{03}$ is goofy, then $l_{02}+l_{03}=l_{02}+l_{13}=0 \bmod l_{03}$, and so $l_{02}=0 \bmod l_{03}$. Either way, since these are all natural numbers this implies that $l_{03} \leq l_{02}+l_{23}$. The other claim is proved similarly.

Finally, notice that the labeled graph $G^{\prime}$ obtained from $G$ by exchanging $p_{0}$ with $p_{3}$ and $p_{1}$ with $p_{2}$ also satisfies the assumption of Lemma 6.3. Moreover, if $G^{\prime}$ satisfies the conclusions of the lemma, then $G$ does as well. Therefore, we can replace $G$ by $G^{\prime}$ at any time. Given this symmetry, we only need to consider eight possible cases.

Case Ia. $\mathbf{e}_{01}$ is the longest edge and it is regular.

Since $e_{01}$ is the longest edge, $l_{01}$ is greater than or equal to $l_{12}, l_{02}, l_{13}$, and $l_{03}$. Since $e_{01}$ is regular, $l_{03}=l_{13} \bmod l_{01}$ and $l_{02}=l_{12} \bmod l_{01}$. Since these are all natural numbers this implies that

$$
l_{03}=l_{13} \quad \text { and } \quad l_{02}=l_{12} .
$$

By Lemma 6.5, the displayed equations imply that (a) is true.

Case $I b . \mathbf{e}_{01}$ is the longest and is goofy.

By an argument similar to the first paragraph above,

$$
l_{02}=l_{13} \quad \text { and } \quad l_{03}=l_{12} .
$$

Hence, (c) is true.

Case IIa. $\mathbf{e}_{12}$ is the longest and is regular.

By an argument similar to the first paragraph of case Ia,

$$
l_{01}=l_{02} \quad \text { and } \quad l_{13}=l_{23} .
$$

Since $l_{01}=l_{02}$, the edge $e_{03}$ is both goofy and regular. Thus $l_{01}+l_{13}=0 \bmod l_{03}$, and hence

$$
l_{03} \leq l_{01}+l_{13} .
$$

Together, the displayed equations imply that (a) is true.

Case IIb. $\mathbf{e}_{\mathbf{1 2}}$ is the longest and is goofy (and not regular).

If $l_{02}=l_{13}$, then since $e_{12}$ is the longest edge, (c) is true; so assume that $l_{02} \neq l_{13}$.

Since $e_{12}$ is the longest edge, $l_{12}$ is strictly greater than $l_{01}$ and $l_{23}$ and greater than or equal to $l_{02}$ and $l_{13}$. Since $e_{12}$ is goofy, $l_{01}+l_{23}=0 \bmod l_{12}$ and $l_{02}+l_{13}=$ $0 \bmod l_{12}$. Since these are all natural numbers and $l_{02} \neq l_{13}$, this implies that

$$
l_{12}=l_{01}+l_{23} \quad \text { and } \quad l_{12}=l_{02}+l_{13} .
$$

Assume first that $e_{01}$ is longer than $e_{23}, e_{02}$, and $e_{13}$. Because $e_{12}$ is not regular, $l_{01} \neq l_{02}$, so this implies that $l_{02}<l_{01}, l_{23} \leq l_{01}$, and $l_{13} \leq l_{01}$. Since also $l_{03} \leq l_{12}=l_{02}+l_{13}$, we conclude that $l_{03}<l_{01}+l_{13}$. If $e_{01}$ is regular, then $l_{03}=l_{13} \bmod l_{01}$, and so $l_{03}=l_{13}$. Since $e_{12}$ is the longest edge, by Lemma 6.5 this 
implies that (a) is true. On the other hand, if $e_{01}$ is goofy, then $l_{02}=l_{13} \bmod l_{01}$, which contradicts $l_{02} \neq l_{13}$.

Up to symmetry, the only remaining possibility is that $e_{02}$ is longer than $e_{01}$, $e_{23}$, and $e_{13}$. This implies that $l_{01}<l_{02}, l_{23}<l_{02}$, and $l_{13} \leq l_{02}$. Since also $l_{03} \leq l_{12}=l_{01}+l_{23}$, we conclude that $l_{03}<l_{02}+l_{23}$. If $e_{02}$ is regular, then $l_{03}=l_{23} \bmod l_{02}$, and so $l_{03}=l_{23}$. Since $e_{12}$ is the longest edge, by Lemma 6.5 this implies that (a) is true. On the other hand, if $e_{02}$ is goofy, then $l_{01}=l_{23} \bmod l_{02}$, and so $l_{01}=l_{23}$. Since $e_{12}$ is the longest edge, this implies that (b) is true.

Case IIIa. $\mathbf{e}_{02}$ is the longest and is regular.

Since $e_{02}$ is the longest edge, $l_{02}$ is strictly greater than $l_{01}, l_{23}$, and $l_{12}$, and greater than or equal to $l_{03}$. Therefore, since $e_{02}$ is regular,

$$
l_{02}=l_{01}+l_{12} \quad \text { and } \quad l_{03}=l_{23} .
$$

By Lemma 6.5. this implies that

$$
l_{03} \leq l_{01}+l_{13} .
$$

Finally, since $l_{03}=l_{23}, e_{13}$ is regular and goofy. Thus, $l_{12}+l_{23}=0 \bmod l_{13}$, and so

$$
l_{13} \leq l_{12}+l_{23} .
$$

Together, the displayed equations imply that (a) is true.

Case IIIb. $\mathbf{e}_{\mathbf{0 2}}$ is the longest and is goofy.

Since $e_{02}$ is the longest edge, $l_{02}$ is strictly greater than $l_{01}, l_{23}$, and $l_{12}$, and greater than or equal to $l_{03}$. Therefore, since $e_{02}$ is goofy,

$$
l_{01}=l_{23} \quad \text { and } \quad l_{02}=l_{03}+l_{12} .
$$

Since $l_{01}=l_{23}$, the edge $e_{13}$ is goofy, that is, $l_{03}+l_{12}=0 \bmod l_{13}$. Hence,

$$
l_{13} \leq l_{03}+l_{12} .
$$

Assume first that $e_{03}$ is regular. Then $l_{03}+l_{12}+l_{01}=l_{02}+l_{23}=\bmod l_{03}$, that is, $l_{01}+l_{12}=0 \bmod l_{03}$. Hence, $l_{03} \leq l_{01}+l_{12}$. Together with the displayed equations above, this implies that (b) is true.

So assume instead that $e_{03}$ is goofy. Then $2 l_{01}=l_{01}+l_{23}=0 \bmod l_{03}$. If $l_{03} \leq l_{01}$ then (b) is true; otherwise, $l_{03}=2 l_{01}=l_{01}+l_{23}$. If $e_{23}$ is regular, then $l_{03}+l_{12}=l_{02}=l_{03} \bmod l_{23}$, while if $e_{23}$ is goofy, then $2 l_{23}=l_{03}=l_{12} \bmod l_{23}$. In either case, $l_{12}=0 \bmod l_{23}$ and so $l_{23} \leq l_{12}$; hence $l_{03} \leq l_{01}+l_{12}$. As before, this implies that (b) is true.

Case IVa. $\mathbf{e}_{\mathbf{0 3}}$ is the longest and is regular.

Since $l_{03}$ is strictly greater than every other $l_{i j}$ and $e_{03}$ is regular,

$$
l_{03}=l_{01}+l_{13}=l_{02}+l_{23} .
$$

Since $l_{03}=l_{02}+l_{23}=l_{23} \bmod l_{02}$, the edge $e_{02}$ is regular. Therefore, $l_{01}+l_{12}=$ $0 \bmod l_{02}$. A similar argument shows $l_{12}+l_{23}=0 \bmod l_{13}$. Therefore,

$$
l_{02} \leq l_{01}+l_{12} \quad \text { and } \quad l_{13} \leq l_{12}+l_{23} .
$$

Together, the displayed equations above imply that (a) is true. 
Case $I V b . \mathbf{e}_{03}$ is the longest and is goofy (and not regular).

Since $l_{03}$ is strictly greater than every other $l_{i j}$ and $e_{03}$ is goofy,

$$
l_{03}=l_{01}+l_{23}=l_{02}+l_{13} .
$$

Assume first that $e_{01}$ is longer than $e_{23}, e_{02}$, and $e_{13}$. If $e_{01}$ is regular, then $l_{01}+l_{23}=l_{03}=l_{13} \bmod l_{01}$, which implies that $l_{13}=l_{23}$. This contradicts the claim that $e_{03}$ is not regular. Therefore, $e_{01}$ is goofy and so $l_{02}=l_{13} \bmod l_{01}$ and $l_{03}=l_{12} \bmod l_{01}$. Since $l_{23}, l_{02}$, and $l_{13}$ are all less than or equal to $l_{01}$ and $l_{12}<l_{03}=l_{01}+l_{23}$, this implies that $l_{02}=l_{13}$ and $l_{12}=l_{23}$. Now if $e_{02}$ is regular, then $2 l_{02}=l_{03}=-l_{23} \bmod l_{02}$, while if $e_{02}$ is goofy, then $2 l_{02}=l_{03}=l_{23} \bmod l_{02}$; either way, $l_{23}=0 \bmod l_{02}$ and so $l_{02} \leq l_{23}$. Since $l_{02} \leq l_{01}$ and $2 l_{02}=l_{01}+l_{23}$, this implies that $l_{01}=l_{02}$, which contradicts the assumption that $e_{03}$ is not regular.

Up to symmetry, the only remaining possibility is that $e_{02}$ is longer than $e_{01}$, $e_{23}$, and $e_{13}$. If $e_{02}$ is regular, then $l_{13}+l_{02}=l_{03}=l_{23} \bmod l_{02}$, which implies that $l_{13}=l_{23}$. This contradicts the assumption that $e_{03}$ is not regular. Therefore $e_{02}$ is goofy and so $l_{01}=l_{23} \bmod l_{02}$; hence, $l_{01}=l_{23}$. If $e_{01}$ is regular, then $2 l_{01}=l_{03}=l_{13} \bmod l_{01}$, that is, $l_{13}=0 \bmod l_{01}$. Since $l_{13}<l_{03}=2 l_{01}$ and $l_{01}=l_{23}$, this implies that $l_{13}=l_{23}$. This contradicts the assumption that $e_{03}$ is not regular. Therefore $e_{01}$ is goofy and so $2 l_{01}=l_{03}=l_{12} \bmod l_{01}$, that is, $l_{12}=0 \bmod l_{01}$. Therefore, $l_{23}=l_{01} \leq l_{12}$. Together with the displayed equations above, this implies that (b) is true.

This completes the proof of Lemma 6.3.

\section{The CASE that The FiXed SET IS Discrete BUt The ASsociated MULTIGRAPH IS NOT SIMPLE}

In this section, we prove Theorem 2 in the case that the fixed set is discrete but the associated multigraph is not simple, that is, it contains multiple edges. In fact, in this case only the last two possibilities can arise.

Proposition 7.1. Let the circle act faithfully on a 6-dimensional compact symplectic manifold $(M, \omega)$ with moment map $\Phi: M \longrightarrow \mathbb{R}$. Assume that $H^{2}(M, \mathbb{R})=\mathbb{R}$, the fixed set is discrete, and the associated multigraph is not simple. Then one of the following two statements is true:

(C) The fixed set consists of four points; the weights at these points are

$$
\{1,2,3\},\{1,-1,4\},\{1,-1,-4\} \text {, and }\{-1,-2,-3\} \text {. }
$$

(D) The fixed set consists of four points; the weights at these points are

$$
\{1,2,3\},\{1,-1,5\},\{1,-1,-5\} \text {, and }\{-1,-2,-3\} \text {. }
$$

Remark 7.2. Every isotropy sphere $N \subset M^{\mathbb{Z}_{k}}$ must contain exactly one fixed point with weight $-k$ and one with weight $k$. Therefore, the manifolds described above must contain a pair of isotropy spheres $N \subset M^{\mathbb{Z}_{2}}$ and $N^{\prime} \subset M^{\mathbb{Z}_{3}}$ which intersect in two points.

Given a labeled multigraph $G$ with vertices $\left\{p_{0}, p_{1}, p_{2}, p_{3}\right\}$, we adopt the following notational conventions: For any pair $\{i, j\} \subset\{0,1,2,3\}, E_{i j}$ is the set of edges from $p_{i}$ to $p_{j}$. Moreover, the edges $e_{i j}$ and $e_{i j}^{\prime}$ (if they exist) lie in $E_{i j}$ and have lengths $l_{i j}$ and $l_{i j}^{\prime}$, respectively. Our proof relies heavily on the following technical lemma. 
Lemma 7.3. Let $G$ be a labeled multigraph with vertices $\left\{p_{0}, p_{1}, p_{2}, p_{3}\right\}$. Assume that the following hold:

(1) The graph is not simple and $l_{e} \neq 1$ for every edge $e$.

(2) The set $\bigcup_{j<i} E_{i j}$ contains at most $i$ edges and the set $\bigcup_{j>i} E_{i j}$ contains at most $3-i$ edges for each vertex $p_{i}$; let the weights at $p_{i}$ be the multiset of $i$ negative integers and $3-i$ positive integers obtained by adding 1 and -1 with appropriate multiplicity to

$$
\left\{\operatorname{sign}(j-i) l_{e} \mid 0 \leq j \leq 3 \text { and } e \in E_{i j}\right\} .
$$

(3) Given any edge $e_{i j}$, the weights at $p_{i}$ and $p_{j}$ agree modulo $l_{i j}$.

(4) Given any distinct pair of edges $e_{i j}$ and $e_{i j}^{\prime}$ in $E_{i j}$, the lengths $l_{i j}$ and $l_{i j}^{\prime}$ are relatively prime.

Then, after possibly exchanging $p_{0}$ with $p_{3}$ and $p_{1}$ with $p_{2}$, one of the following three statements is true:

(x) $E$ is either $\left\{e_{03}, e_{03}^{\prime}\right\}$ or $\left\{e_{03}, e_{03}^{\prime}, e_{12}\right\}, l_{03}=3$, and $l_{03}^{\prime}=2$.

(y) $E=\left\{e_{12}, e_{12}^{\prime}, e_{01}, e_{23}, e_{03}\right\}$ and $l_{12}^{\prime}=l_{01}=l_{23}=l_{03}=2$.

(z) $E=\left\{e_{03}, e_{02}, e_{02}^{\prime}, e_{23}\right\}, l_{03}=4, l_{02}=3$, and $l_{02}^{\prime}=l_{23}=2$.

Proof of Proposition 7.1. By the first paragraph of the proof of Proposition 6.1, there is exactly one fixed point $p_{i}$ of index $2 i$ for all $i$ such that $0 \leq 2 i \leq 6$ and $\Phi\left(p_{i}\right)<\Phi\left(p_{j}\right)$ exactly if $i<j$.

We will now check that the associated labeled multigraph $G$ satisfies the assumptions of Lemma 7.3. Since $G$ is not simple, it satisfies assumption (1). By Lemma 5.3. assumption (2) holds and the weights at $p_{i}$ consist of the multiset of $i$ negative integers and $3-i$ positive integers obtained by adding 1 and -1 with appropriate multiplicity to $\left\{\operatorname{sign}(j-i) l_{e} \mid 0 \leq j \leq 3\right.$ and $\left.e \in E_{i j}\right\}$. Finally, assumptions (3) and (4) hold by Lemmas 5.4 and 5.5, respectively. Therefore, Lemma 7.3 implies that one of the statements $(\mathrm{x}),(\mathrm{y})$, or $(\mathrm{z})$ is true.

First assume that statement $(\mathrm{x})$ is true. For some natural number $l_{12} \geq 1$, the weights at $p_{0}, p_{1}, p_{2}$, and $p_{3}$ are $\{1,2,3\},\left\{1,-1, l_{12}\right\},\left\{1,-1,-l_{12}\right\}$, and $\{-1,-2,-3\}$, respectively. By Proposition 3.9 (see also Example 3.15), as an $H^{*}\left(\mathbb{C P}^{\infty} ; \mathbb{Z}\right)=\mathbb{Z}[t]$ module, $H_{S^{1}}^{*}(M ; \mathbb{Z})$ is generated by $1, \alpha_{1}, \alpha_{2}, \alpha_{3}$, where

$$
\begin{gathered}
\left.\alpha_{1}\right|_{p_{1}}=t,\left.\alpha_{1}\right|_{p_{2}}=\frac{6+l_{12}}{6-l_{12}} t,\left.\alpha_{1}\right|_{p_{3}}=\frac{12}{6-l_{12}} t, \\
\left.\alpha_{2}\right|_{p_{2}}=l_{12} t^{2},\left.\alpha_{2}\right|_{p_{3}}=6 t^{2},\left.\alpha_{3}\right|_{p_{3}}=6 t^{3}, \text { and }\left.\alpha_{i}\right|_{p_{j}}=0 \quad \forall j<i .
\end{gathered}
$$

Since $\alpha_{1}$ is an integral class, this implies that $6-l_{12}$ divides 12 . In particular, $l_{12} \neq 1$ and so there is an isotropy sphere $N \subset M^{\mathbb{Z}_{l_{12}}}$ which contains $p_{1}$ and $p_{2}$. Let $\gamma \in H_{S^{1}}^{4}(M ; \mathbb{Z})$ be the push-forward in equivariant cohomology of the natural generator $1 \in H_{S^{1}}^{0}(N)$. Since $p_{0}$ and $p_{3}$ are not in $N,\left.\gamma\right|_{p_{0}}=\left.\gamma\right|_{p_{3}}=0$. On the other hand, for $i=1$ or $2,\left.\gamma\right|_{p_{i}}$ is the product of the weights of the normal bundle to $N$ at $p_{i}$, that is, $\left.\gamma\right|_{p_{1}}=\left.\gamma\right|_{p_{2}}=-t^{2}$. Comparing with equation (7.4), this implies that $\gamma=\frac{2}{6-l_{12}} \alpha_{2}-\alpha_{1} t$. Since $\gamma$ is an integral linear combination of $t^{2}, \alpha_{1} t$, and $\alpha_{2}$, this implies that $6-l_{12}$ divides 2 . Hence, $l_{12}=4,5,7$ or 8 . Finally, Lemma 3.23 implies that $6=\Gamma_{p_{0}}>\Gamma_{p_{1}}=l_{12}$. Hence, $l_{12}=4$ or 5 , as required.

Now assume that statement (y) is true. Then the weights at the fixed points are $\{1,2,2\},\left\{2,-2, l_{12}\right\},\left\{2,-2,-l_{12}\right\}$ and $\{-1,-2,-2\}$ for some natural number $l_{12}$. Since $\int_{M} c_{1}^{S^{1}}(M)=0$, Theorem 2.4 (together with Remark 2.5) implies that $\frac{5}{4}-\frac{1}{4}-\frac{1}{4}+\frac{5}{4}=0$, which is not true. 
Finally, assume that statement $(\mathrm{z})$ holds. Then the weights at the fixed points are either

- $\{2,3,4\},\{1,1,-1\},\{2,-2,-3\}$, and $\{-1,-2,-4\}$; or

- $\{-2,-3,-4\},\{-1,-1,1\},\{-2,2,3\}$, and $\{1,2,4\}$.

Since $\int_{M} 1=0$, Theorem 2.4 implies that $\frac{1}{24}-1+\frac{1}{12}-\frac{1}{8}=0$, which is not true.

We will spend the remainder of this section proving Lemma 7.3. Let $G$ be any labeled multigraph satisfying the assumptions of the lemma.

Consider any distinct pair of edges $e_{i j}$ and $e_{i j}^{\prime}$ in $E_{i j}$, where $i<j$. By assumption (4), the lengths $l_{i j}$ and $l_{i j}^{\prime}$ are relatively prime. By definition, the weights at $p_{i}$ and $p_{j}$ are $\left\{l_{i j}, l_{i j}^{\prime}, x\right\}$ and $\left\{-l_{i j},-l_{i j}^{\prime}, y\right\}$, respectively, for some integers $x$ and $y$. By assumption (3), these sets agree modulo $l_{i j}$ and also agree modulo $l_{i j}^{\prime}$. Therefore, we can apply the following facts.

Lemma 7.5. Let $l \geq l^{\prime} \geq 1$ be relatively prime natural numbers; let $x$ and $y$ be integers. Assume that the multisets $\left\{l, l^{\prime}, x\right\}$ and $\left\{-l,-l^{\prime}, y\right\}$ are equal modulo $l$ and also equal modulo $l^{\prime}$. Then the following hold:

(i) If $l^{\prime} \neq 1$, then $2 l^{\prime} \neq 0 \bmod l, x \neq y \bmod l, x+l^{\prime}=0 \bmod l$, and $y=l^{\prime} \bmod l$.

(ii) If $l \geq x>0$, then either $l^{\prime}=2=l-x$ and $x$ and $y$ are odd, $l^{\prime}=1=l-x$, or $l^{\prime}=1$ and $x=l$. A similar claim holds if $l \geq-y>0$.

(iii) If $l \geq y>0$, then either $l^{\prime}=2=y$ and $l$ is odd, $l^{\prime}=1=y$, or $l^{\prime}=1$ and $l=2=y$. A similar claim holds if $l \geq-x>0$.

Proof. If $2 l^{\prime}=0 \bmod l$, then since $l$ and $l^{\prime}$ are relatively prime and $l \geq l^{\prime}, l^{\prime}=1$ and $l \leq 2$. Otherwise, since $2 l=0 \bmod l$, the assumption that $\left\{l, l^{\prime}, x\right\}$ and $\left\{-l,-l^{\prime}, y\right\}$ are equal modulo $l$ implies immediately that $2 l^{\prime} \neq 0 \bmod l, x \neq y \bmod l$, $x+l^{\prime}=0 \bmod l$, and $y=l^{\prime} \bmod l$. In particular, (i) holds. Similarly, if $l^{\prime}>2$, then $2 l \neq 0 \bmod l^{\prime}$ and so $l=y \bmod l^{\prime}$ and $l+x=0 \bmod l^{\prime}$.

To prove (ii), assume that $l \geq x>0$. If $l^{\prime}=1$ and $l \leq 2$, then $l \geq x>0$ implies immediately that either $l^{\prime}=l-x$ or $x=l$. Otherwise, $x+l^{\prime}=0 \bmod l$ by the previous paragraph, and $l>l^{\prime}$ since $l$ and $l^{\prime}$ are relatively prime. Therefore, $l^{\prime}=l-x$. If $l^{\prime}>2$, then $2 l \neq 0 \bmod l^{\prime}$ and $l+x=0 \bmod l^{\prime}$, that is, $2 l=0 \bmod l^{\prime}$. Since these equations give a contradiction, $l^{\prime} \leq 2$, as required. Finally, if $l^{\prime}=2$, then $l$ is odd since $l$ and $l^{\prime}$ are relatively prime. Since $x=l-2, x$ is odd as well. Moreover, since $2 l=0 \bmod 2, x+y=0 \bmod 2$, and so $y$ is also odd.

To prove (iii), assume that $l \geq y>0$. If $l^{\prime}=1$ and $l \leq 2$, then $l \geq y>0$ implies immediately that either $y=1$ or $l=2=y$. Otherwise, by the first paragraph, $y=l^{\prime} \bmod l$, and so $y=l^{\prime}$. If $l^{\prime}>2$, then $l=y \bmod l^{\prime}$, that is, $l=0 \bmod l^{\prime}$. Since this is impossible, $l^{\prime} \leq 2$, as required. Finally, if $l^{\prime}=2$, then $l$ is odd since $l$ and $l^{\prime}$ are relatively prime.

Finally, notice that the labeled multigraph $G^{\prime}$ obtained by exchanging $p_{0}$ with $p_{3}$ and $p_{1}$ with $p_{2}$ also satisfies the assumptions of Lemma 7.3. Therefore, we can replace $G$ by $G^{\prime}$ at any time. Given this symmetry, we only need to consider four cases.

Case I. $\mathbf{E}_{\mathbf{0 2}} \neq \emptyset$ and $\mathbf{E}_{\mathbf{0 3}}$ contains at least two edges.

Let $l_{03} \geq l_{03}^{\prime} \neq 1$ be the lengths of two edges $e_{03}$ and $e_{03}^{\prime}$ from $p_{0}$ to $p_{3}$, and let $l_{02}$ be the length of an edge $e_{02}$ from $p_{0}$ to $p_{2}$. Let $m$ be the positive weight at $p_{2}$. 
Note that since $l_{03}^{\prime} \neq 1$, (ii) above implies that the third weight at $p_{0}$ cannot be either $l_{03}$ or $l_{03}^{\prime}$. Hence, there cannot be any other edge of length $l_{03}$ or $l_{03}^{\prime}$ with minimum $p_{0}$. A similar argument proves that there cannot be any other edge of length $l_{03}$ or $l_{03}^{\prime}$ with maximum $p_{3}$. In particular, if $m$ is $l_{03}$ or $l_{03}^{\prime}$, then since $m>1$, there is an edge from $p_{2}$ to $p_{3}$ with length $l_{03}$ or $l_{03}^{\prime}$; this is impossible.

Suppose first that $l_{02} \geq l_{03}$ and $l_{02} \geq m$. Then compatibility along the edge $e_{02}$ implies that $m$ is either $l_{03}$ or $l_{03}^{\prime}$. As we have seen above, this is impossible.

So suppose next that $m \geq l_{03}$ and $m \geq l_{02}$. Since $m \neq 1$, there is an edge $e_{23} \in E_{23}$ of length $m$. Compatibility along $e_{23}$ implies that $l_{02}$ is either $l_{03}$ or $l_{03}^{\prime}$. Again, this is impossible.

Finally, suppose that $l_{03}>l_{02}$ and $l_{03}>m$. Since $l_{03}^{\prime} \neq 1$, by (ii) above $l_{03}^{\prime}=2$ and $l_{03}=l_{03}^{\prime}+l_{02}$. But then compatibility along $e_{02}$ implies that $m=l_{03}^{\prime} \bmod l_{02}$. Because $l_{03}^{\prime}+l_{02}=l_{03}>m$ and $l_{02} \geq l_{03}^{\prime}$ (since $l_{02} \neq 1$ and $l_{03}^{\prime}=2$ ), this implies that $m=l_{03}^{\prime}$. Once again, this is impossible.

Case J. $\mathbf{E}_{\mathbf{0 2}} \neq \emptyset$ and $\mathbf{E}_{\mathbf{0 3}}$ contains exactly one edge.

By assumptions (11) and (2), this implies that - up to symmetry - there must be exactly two edges $e_{02}$ and $e_{02}^{\prime}$ from $p_{0}$ to $p_{2}$; we may assume that $l_{02} \geq l_{02}$. Let $l_{03}$ denote the length of the edge $e_{03}$ from $p_{0}$ to $p_{3}$. Since $l_{02}^{\prime} \neq 1$, by (iii) above the positive weight at $p_{2}$ is not 1 . Hence, the graph also contains an edge $e_{23}$ of length $l_{23}$ from $p_{2}$ to $p_{3}$. By assumption (2), after possibly adding an edge of length 1 , the graph consists of these edges and an edge $e_{13}$ of length $l_{13}$ from $p_{1}$ to $p_{3}$.

Assume first that $l_{02} \geq l_{03}$ and $l_{02} \geq l_{23}$. Since $l_{02}^{\prime} \neq 1$, by (ii) and (iii) above this implies that $l_{23}$ is both even and odd, which is impossible.

Now, assume that that $l_{23} \geq l_{03}$ and $l_{23} \geq l_{02}$. Then compatibility along $e_{23}$ implies that $l_{03}$ is either equal to $l_{02}$ or $l_{02}^{\prime}$. Since $l_{02}^{\prime} \neq 1$, this contradicts (ii) above, just as in the previous case.

Now, assume that $l_{13} \geq l_{03}$ and $l_{13} \geq l_{23}$. Since the negative weight at $p_{1}$ is -1 , compatibility along $e_{13}$ implies that $l_{03}=1$ or $l_{23}=1$. This is impossible.

Finally, assume that $l_{03}>l_{02}, l_{03}>l_{23}$, and $l_{03} \geq l_{13}$. Then compatibility along $e_{03}$ implies that either $l_{03}=l_{23}+l_{02}^{\prime}=l_{13}+l_{02}$ or $l_{03}=l_{23}+l_{02}=l_{13}+l_{02}^{\prime}$. Since $l_{02}^{\prime} \neq 1$, fact (i) above implies that $l_{03} \neq l_{23} \bmod l_{02}$. Hence,

$$
l_{03}=l_{23}+l_{02}^{\prime}=l_{13}+l_{02} \text {. }
$$

But (i) above also implies that $l_{03}+l_{02}^{\prime}=0 \bmod l_{02}$, and $l_{23}=l_{02}^{\prime} \bmod l_{02}$. Hence, $3 l_{02}^{\prime}=0 \bmod l_{02}$. Since $l_{02}$ and $l_{02}^{\prime}$ are relatively prime and $l_{02} \geq l_{02}^{\prime} \neq 1$, this implies that

$$
l_{02}=3 \quad \text { and } \quad l_{02}^{\prime}=2 .
$$

Since $l_{03}=l_{02}^{\prime} \bmod l_{23}$, compatibility along $e_{23}$ implies that $l_{02}=l_{13} \bmod l_{23}$; hence $4=0 \bmod l_{23}$. Moreover, $l_{23}=l_{02}^{\prime} \bmod l_{02}$, that is, $l_{23}=2 \bmod 3$. Combined, these imply that

$$
l_{23}=2 .
$$

Together, the displayed equations imply that $(\mathrm{z})$ is true.

Case K. $\mathbf{E}_{02} \neq \emptyset$ and $\mathbf{E}_{03}=\emptyset$.

Since $E_{02} \neq \emptyset$, assumption (2) implies that $E_{12}$ cannot contain two edges. By assumption (1), this implies that either $E_{02}$ or $E_{13}$ must contain two edges. Hence, by assumption (2), after possibly adding edges of length 1 , the multigraph contains 
exactly six edges: $e_{02}, e_{02}^{\prime}, e_{13}, e_{13}^{\prime}, e_{01}$ and $e_{23}$. We may assume that $l_{13} \geq l_{13}^{\prime}$ and that $l_{02} \geq l_{02}^{\prime}$. By assumption (1), we cannot have $l_{02}^{\prime}=l_{13}^{\prime}=1$.

Assume first that $l_{01} \geq l_{02}$ and $l_{01} \geq l_{13}$. Then compatibility along $e_{01}$ implies that $l_{02}=l_{13}$ and $l_{02}^{\prime}=l_{13}^{\prime}$; hence $l_{02}^{\prime}=l_{13}^{\prime} \neq 1$. Since $l_{02}^{\prime} \neq 1$, (i) above implies that $2 l_{02}^{\prime} \neq 0 \bmod l_{02}$ and $l_{02}^{\prime}=l_{23} \bmod l_{02}$. Since $l_{13}^{\prime} \neq 1$, (i) above implies that $l_{13^{\prime}}+l_{23}=0 \bmod l_{13}$, that is, $l_{02}^{\prime}+l_{23}=0 \bmod l_{02}$. Together, these three equations give a contradiction.

Hence, by symmetry we may assume that $l_{02}>l_{01}, l_{02}>l_{23}$, and $l_{02} \geq l_{13}$. If $l_{02}^{\prime} \neq 1$, (ii) and (iii) above imply that $l_{23}$ is both odd and even. Since this is impossible, $l_{02}^{\prime}=1$. Therefore, (ii) above implies that $l_{02}=l_{01}+1$. Now, since $l_{02} \geq l_{13} \geq l_{13}^{\prime}$ and $l_{13}$ and $l_{13}^{\prime}$ are relatively prime, compatibility along $e_{01}$ implies that $l_{13}^{\prime}=l_{02}^{\prime}=1$, which is impossible.

Case L. $\mathbf{E}_{02}=\mathbf{E}_{13}=\emptyset$.

First suppose that there are three edges from $p_{0}$ to $p_{3}$ of length $l_{03} \geq l_{03}^{\prime} \geq l_{03}^{\prime \prime}>$ 1 . Then by (ii) above $l_{03}^{\prime} \leq 2$, which is impossible. So there are at most two edges from $p_{0}$ to $p_{3}$.

Therefore, by assumption (2), after possibly adding edges of length 1, $G$ contains exactly six edges: $e_{03}, e_{03}^{\prime}, e_{12}, e_{12}^{\prime}, e_{01}$, and $e_{23}$. We may assume that $l_{03} \geq l_{03}^{\prime}$ and that $l_{12} \geq l_{12}^{\prime}$. By symmetry, we may also assume that $l_{01} \geq l_{23}$. By assumption (1), we cannot have $l_{03}^{\prime}=l_{12}^{\prime}=1$.

First, assume that $l_{01} \geq l_{03}$ and $l_{01} \geq l_{12}$. Then compatibility along $e_{01}$ implies that $l_{03}=l_{12}$ and $l_{03}^{\prime}=l_{12}^{\prime}$; hence $l_{03}^{\prime}=l_{12}^{\prime} \neq 1$. Since $l_{03}^{\prime} \neq 1$, (i) above implies that $2 l_{03}^{\prime} \neq 0 \bmod l_{03}$ and $l_{03}^{\prime}+l_{01}=0 \bmod l_{03}$. Since $l_{12}^{\prime} \neq 1$, (i) above implies that $l_{12}^{\prime}=l_{01} \bmod l_{12}$, that is, $l_{03}^{\prime}=l_{01} \bmod l_{03}$. Together, these three equations give a contradiction.

Next, assume that $l_{12} \geq l_{01} \geq l_{03}$. Since $l_{12} \geq l_{01} \geq l_{23}$, by (iii) above either $l_{12}^{\prime}=l_{01}=l_{23}=2$ and $l_{12}$ is odd, or $l_{12}^{\prime}=1$. In the first case, compatibility along $e_{01}$ implies that $l_{03}=2$ and $l_{03}^{\prime}=1$; therefore, statement (y) holds. In the second case, compatibility along $e_{01}$ implies that $l_{03}^{\prime}=1$, which is impossible.

Next, assume that $l_{03}>l_{01}>l_{12}$. Then, since $l_{03}>l_{01}$, by (ii) above, $2 \geq$ $l_{03}^{\prime}=l_{03}-l_{01}$. Since $l_{01} \neq 1$, this implies that $l_{01} \geq l_{03}^{\prime}$. So compatibility along $e_{01}$ implies that $l_{12}=l_{12}^{\prime}=l_{03}^{\prime}$. Since $l_{12}$ and $l_{12}^{\prime}$ are relatively prime this implies that $l_{12}^{\prime}=l_{03}^{\prime}=1$, which is impossible.

Finally, assume that $l_{12} \geq l_{01}$ and $l_{03}>l_{01}$. Since $l_{03}>l_{01}$, by (ii) above either $l_{03}^{\prime}=l_{03}-l_{01}=2$ and $l_{01}$ is odd, or $l_{03}^{\prime}=l_{03}-l_{01}=1$. In the first case, since $l_{12} \geq l_{01}$ and $l_{01}$ is odd, by (iii) above, $l_{12}^{\prime}=l_{01}=1$. Since $l_{01} \geq l_{23}$ and $l_{03}=l_{01}+2$, this implies that $l_{23}=1$ and $l_{03}=3$ as well; therefore, statement $(\mathrm{x})$ holds. In the second case, since $l_{12} \geq l_{01}$ and $l_{12}^{\prime} \neq 1$, by (iii) above, $l_{12}^{\prime}=l_{01}=2$, and so $l_{03}=l_{01}+1=3$. But then $l_{03}$ and $l_{03}^{\prime}$ are both odd, while $l_{12}^{\prime}$ is even. This violates compatibility along $e_{01}$.

This completes the proof of Lemma 7.3 .

\section{REFERENCES}

[AB] M. Atiyah and R. Bott, The moment map and equivariant cohomology. Topology 23 (1984) 1-28. MR721448 (85e:58041)

[BV] N. Berline and M. Vergne, Classes caractéristiques équivariantes, formule de localisation en cohomologie équivariante, C. R. Acad. Sci. Paris 295 (1982) 539-541. MR685019 (83m:58002) 
[Dej] I. J. Dejter, Smooth $S^{1}$ manifolds in the homotopy type of $\mathbb{C P}^{3}$, Michigan Math. J. 23 (1976), 83-95. MR0402789 (53:6603)

[Del] T. Delzant, Hamiltoniens périodiques et images convexes de l'application moment, Bull. Soc. Math. France 116 (1998) 315-339. MR.984900 (90b:58069)

[Des] A. Dessai, Homotopy complex projective spaces with Pin(2)-action, Topology and its Applications, Volume 122, Number 3 (2002), 487-499. MR1911696 (2003f:58048)

[GGK] V. Ginzburg, V. Guillemin, and Y. Karshon, Moment maps, cobordisms, and Hamiltonian group actions, Mathematical Surveys and Monographs, Vol. 98, American Mathematical Society, 2002. MR1929136 (2003m:53149)

[Go] L. Godinho, Blowing up Symplectic Orbifolds, Annals of Global Analysis and Geometry 20: 117-162, 2001. MR1857175 (2002k:53162)

[Ha] A. Hattori, Spinc-Structures and $S^{1}$-Actions, Invent. Math. 48 (1978), 7-31. MR508087 (80e:57051)

[HBJ] F. Hirzebruch, T. Berger, and R. Jung, Manifolds and modular forms, Aspects of Mathematics, E20, Braunschweig, (1992). MR1189136 (94d:57001)

[Hu $\quad$ D. Husemöller, Fibre bundles, Springer, 1994. MR1249482 (94k:55001)

[Ja] D. M. James, Smooth $S^{1}$-actions on homotopy $\mathbb{C P}^{4}$ 's, Michigan Math. J. 32 (1985), 259-266. MR803831 (87c:57031)

[Kar] Y. Karshon, Periodic Hamiltonian flows on four dimensional manifolds, Memoirs Amer. Math. Soc. 141 (1999). MR.1612833 (2000c:53113)

[Ki] F. Kirwan, Cohomology of Quotients in Symplectic and Algebraic Geometry, Princeton University Press, 1984. MR766741 (86i:58050)

[Li1] H. Li, $\pi_{1}$ of Hamiltonian $S^{1}$ manifolds, Proc. Amer. Math. Soc. 131 (2003), 3579-3582. MR.1991771 (2004b:53145)

[Li2] H. Li, Semi-free Hamiltonian circle actions on 6 dimensional symplectic manifolds. Trans. Amer. Math. Soc. 355 (2003) 4543-4568. MR1990761 (2004e:53127)

[Ma] M. Masuda, On smooth $S^{1}$-actions on cohomology projective spaces. The case where the fixed point set consists of four connected components, J. Fac. Sci. Univ. Tokyo 28 (1981), 127-167. MR617869 (82i:57031)

[Mc1] D. McDuff, Symplectic embeddings of 4-dimensional ellipsoids, arXiv:0807.0900.

[Mc2] D. McDuff, Some 6-dimensional Hamiltonian $S^{1}$ manifolds, arXiv:0808.3549.

[Mu] O. R. Muslin, Unitary actions of $S^{1}$ on complex projective spaces, Russian Math. Surveys 33:6 (1978) 249-250. MR526030 (81a:57037)

[Pe1] T. Petrie, Smooth $S^{1}$-actions on cohomology complex projective spaces and related topics, Bull. Math. Soc. 78 (1972), 105-153. MR0296970 (45:6029)

[Pe2] T. Petrie, Torus actions on homotopy complex projective spaces, Invent. Math. 20 (1973), 139-146. MR0322893 (48:1254)

[S] P. Schlenk, Embedding problems in symplectic geometry, De Gruyter Expositions in Mathematics, de Gruyter Verlag, Berlin (2005).

[ToWe1] S. Tolman and J. Weitsman, The cohomology rings of symplectic quotients. Comm. Anal. Geom. 11 (2003), no. 4, 751-773. MR.2015175 (2004k:53140)

[ToWe2] S. Tolman and J. Weitsman, On semifree circle actions with isolated fixed points, Topology, 39 (2000) no. 2, 299-310. MR1722020 (2000k:53074)

[TsWa] E. Tsukada and R. Washiyama, Smooth $S^{1}$-actions on cohomology complex projective spaces with three components of the fixed point set, Hiroshima Math. J. 9 (1979), 41-46. MR.529325 (80j:57043)

[Wal] C. T. C. Wall, Classification problems in differential topology V: On certain 6-manifolds, Invent. Math. 1, 355-374 (1996). MR0216510 (35:7343)

[Wan] K. Wang, Differentiable circle group actions on homotopy complex projective spaces, Math. Ann. 214 (1975), 73-80. MR0372895 (51:9099)

[Yo] T. Yoshida, On smooth semi-free $S^{1}$-actions on cohomology projective spaces, Publ. Res. Inst. Math. Sci. 11 (1976), 483-496. MR0445528 (56:3868)

Department of Mathematics, University of Illinois at Urbana-Champaign, Urbana, ILLINOIS 61801

E-mail address: stolman@math.uiuc.edu 